This item was submitted to Loughborough's Research Repository by the author.

Items in Figshare are protected by copyright, with all rights reserved, unless otherwise indicated.

\title{
Fall-off of eigenfunctions for non-local Schrödinger operators with decaying potentials
}

PLEASE CITE THE PUBLISHED VERSION

http://dx.doi.org/10.1007/s11118-016-9597-3

\section{PUBLISHER}

(C) Springer Science+Business Media Dordrecht

\section{VERSION}

AM (Accepted Manuscript)

\section{PUBLISHER STATEMENT}

This work is made available according to the conditions of the Creative Commons Attribution-NonCommercialNoDerivatives 4.0 International (CC BY-NC-ND 4.0) licence. Full details of this licence are available at: https://creativecommons.org/licenses/by-nc-nd/4.0/

\section{LICENCE}

CC BY-NC-ND 4.0

\section{REPOSITORY RECORD}

Kaleta, Kamil, and Jozsef Lorinczi. 2016. "Fall-off of Eigenfunctions for Non-local Schrödinger Operators with Decaying Potentials". Loughborough University. https://hdl.handle.net/2134/21501. 


\title{
FALL-OFF OF EIGENFUNCTIONS FOR NON-LOCAL SCHRÖDINGER OPERATORS WITH DECAYING POTENTIALS
}

\author{
KAMIL KALETA AND JÓZSEF LŐRINCZI
}

\begin{abstract}
We study the spatial decay of eigenfunctions of non-local Schrödinger operators based on generators of symmetric jump-paring Lévy processes with Kato-class potentials decaying at infinity. This class of processes has the property that the intensity of single large jumps dominates the intensity of all multiple large jumps. We find that the decay rates of eigenfunctions depend on the process via specific preference rates in particular jump scenarios, and depend on the potential through the distance of the corresponding eigenvalue from the edge of the continuous spectrum. We prove that the conditions of the jump-paring class imply that for all eigenvalues the corresponding positive eigenfunctions decay at most as rapidly as the Lévy intensity. This condition is sharp in the sense that if the jump-paring property fails to hold, then eigenfunction decay becomes slower than the decay of the Lévy intensity. We furthermore prove that under reasonable conditions the Lévy intensity also governs the upper bounds of eigenfunctions, and a ground state is comparable to it by two-sided bounds. As an interesting consequence, we identify a sharp regime change in the decay of eigenfunctions as the Lévy intensity is varied from sub-exponential to exponential order, and dependent on the location of the eigenvalue, in the sense that through the transition Lévy intensity-driven decay becomes slower than the rate of Lévy intensity. Our approach is based on path integration and probabilistic potential theory techniques, and all results are also illustrated by specific examples.
\end{abstract}

\section{Introduction}

Non-local Schrödinger operators of the form

$$
H=H_{0}+V,
$$

where $H_{0}$ is a non-local (pseudo-differential, see [30]) operator giving the kinetic term and $V$ is a multiplication operator called potential, receive increasing attention in both pure and applied mathematics. They pose intriguing problems in the intersection areas of functional analysis, PDE and probability, and offer a new framework in scientific modelling, providing life-like correctives and refinements to established theories. In the present paper we are focusing on some spectral properties of such operators by developing a stochastic approach via Feynman-Kac type representations.

There are few examples in which the spectrum and the eigenfunctions of a non-local Schrödinger operator are explicitly known, see [43, 20]. When such detailed expressions are not available, it is natural to ask about the spatial decay properties of eigenfunctions in function of $V$ at least in terms of estimates. A basic interest in this property is that it tells of how well a quantum particle described by $H$ is localized in physical space or, alternatively, of the concentration of mass in the stationary measure of the random process corresponding to $H$.

Key-words: symmetric Lévy process, subordinate Brownian motion, Feynman-Kac semigroup, non-local Schrödinger operator, jump-paring condition, ground state, decay of eigenfunctions, negative eigenvalue, first hitting time of balls

2010 MS Classification: Primary 47D08, 60G51; Secondary 47D03, 47G20

K. Kaleta was supported by the National Science Center (Poland) grant 2012/04/S/ST1/00093 and by the Foundation for Polish Science. We both are grateful to IHÉS, Bures-sur-Yvette, for a research stay where this work has started. 
The pointwise decay of eigenfunctions of Schrödinger operators, i.e., when $H_{0}=-\frac{1}{2} \Delta$ and the underlying random process is Brownian motion, has been much studied in the literature, see e.g. $[1,11,50,51]$ and the references therein. Suppose $\lambda \in \operatorname{Spec} H$ is an isolated eigenvalue and $\varphi \in L^{2}\left(\mathbf{R}^{d}\right)$ is a corresponding eigenfunction of $H$, which is called a ground state and will be denoted by $\varphi_{0}$ when the eigenvalue lies at the bottom of the spectrum, i.e., $\lambda_{0}=\inf \operatorname{Spec} H$. When $V$ is a confining potential in the sense that $V(x) \rightarrow \infty$ as $|x| \rightarrow \infty$, the spectrum of $H$ is purely discrete and a typical answer is that the decay of eigenfunctions is exponential or faster. For instance, if $V(x) \asymp|x|^{2 \beta}, \beta>1$, then the ground state decays super-exponentially like

$$
\varphi_{0}(x) \asymp|x|^{-\frac{1+\beta-d}{2}} e^{-\frac{1}{1+\beta}|x|^{1+\beta}}
$$

i.e., the rate of decay is directly determined by the rate of asymptotic growth of the potential. In this case the semigroup $\left\{e^{-t H}: t \geq 0\right\}$ is intrinsically ultracontractive, and the other eigenfunctions are asymptotically dominated by the ground state $[18,4]$. When the potential is decaying, i.e., $V(x) \rightarrow 0$ as $|x| \rightarrow \infty$, the situation is more delicate. In such cases the discrete component of the spectrum may be empty and thus eigenfunctions may not exist at all. However, if an eigenfunction does exist, then under some further conditions on the potential it can be shown that it still decays exponentially $[1,11]$. A typical result is then that whenever $V \in L_{\text {loc }}^{\infty}\left(\mathbf{R}^{d}\right)$ such that $\liminf _{|x| \rightarrow \infty} V(x) \geq 0$ and $\varphi$ is an eigenfunction for eigenvalue $\lambda<0$, then for every $\varepsilon \in(0,|\lambda|)$ there exists $C_{\varepsilon}>0$ such that

$$
|\varphi(x)| \leq C_{\varepsilon} e^{-\sqrt{\frac{|\lambda|-\varepsilon}{2}}|x|} .
$$

When in $H$ the term $H_{0}$ is replaced by a non-local operator, the behaviours change essentially. In the paper [34] we have considered not just one but a whole class of operators $H_{0}$ corresponding to the generators of symmetric Lévy processes with the property that all multiple large jumps are dominated under the Lévy measure with density $\nu$ by a single large jump (which we call jumpparing Lévy processes, see Section 2.1 below). This class includes important examples such as $H_{0}=\left(-\Delta+m^{2 / \alpha}\right)^{\alpha / 2}-m, 0<\alpha<2$, with polynomially decaying Lévy measure for $m=0$ (isotropic $\alpha$-stable processes, see [33, 32]), and exponentially localized Lévy measure for $m>0$ (relativistic $\alpha$-stable processes), and has a non-trivial overlap with the class of non-local operators obtained under Bernstein functions of the Laplacian [27]. Taking a sufficiently regular Kato-class confining potential $V$, we showed that the ground state of $H$ behaves like

$$
\varphi_{0}(x) \asymp \frac{\nu(x)}{V(x)} .
$$

This gives a neat account of the separate contributions of the unperturbed process and of the perturbation into the decay. For general Kato-class potentials, (1.2) has the generic form

$$
\varphi_{0}(x) \asymp \nu(x) \Lambda_{V}(x),
$$

where $\Lambda_{V}(x)$ is the mean exit time from a unit ball centered at the starting point $x$ of the process under the potential $V$. This formula gives a probabilistic interpretation to the decay of ground states, from which the above estimate is also derived. Moreover, we proved that the other eigenfunctions $\varphi_{n}, n \in \mathbb{N}$, satisfy

$$
\left|\varphi_{n}(x)\right| \leq C_{n} \varphi_{0}(x), \quad x \in \mathbf{R}^{d},
$$

with a suitable constant $C_{n}(X, V)>0$, dependent on the process and the potential. We emphasize that this ground state domination follows in the non-local case with no involvement and also in lack of intrinsic ultracontractivity. For the fractional Laplacian ( $m=0$ above) and the same potential 
$V(x) \asymp|x|^{2 \beta}$ as above, this means now a much slower decay like

$$
\varphi_{0}(x) \asymp \frac{1}{|x|^{\alpha+d+2 \beta}} .
$$

The picture for confining potentials corresponds to the intuition that in this case there is a killing mechanism with increasing values of the potential, and the tails of the ground state should depend on the balance between the potential and the Lévy intensity. In terms of techniques, these results have been established by using sharp uniform estimates on the local extrema of harmonic functions for the perturbed Lévy process combined with a close systematic control of jumps, based on the jump-paring property (see Definition 2.1 below).

Eigenfunction decay for non-local Schrödinger operators with potentials decreasing to zero has been little understood so far. In the seminal paper [12] it was considered for the operators $H_{0}=$ $(-\Delta)^{\alpha / 2}, 0<\alpha<2$, and $H_{0}=\sqrt{-\Delta+m^{2}}-m, m>0$, using martingale and optional stopping methods combined with precise estimates of the corresponding resolvent kernels. For an extension of these ideas to other operators of interest in mathematical physics see [28, 42].

In the present work we use the wide framework of symmetric jump-paring processes introduced in [34], which has the advantage of accommodating a large selection of interesting types of Lévy processes without being too abstract, and focus here on the complementary and essentially different case of potentials decreasing to zero at infinity. Our aim is to derive the fall-off behaviour of eigenfunctions in function of the process and the potential. In particular, a main question we address is if the basic relationship (1.3) continues to hold for decaying potentials. For a potential $V$ decaying to zero the perturbed processes behave far out like free processes, thus we have $\Lambda_{V}(x) \asymp$ const and it can be expected that $\varphi_{0} \asymp \nu$. This means that there is an essential difference from the confining case in that there is no longer a balancing mechanism as now both $\nu$ and $V$ decrease with the distance from the origin. A main consequence is that the contribution of both the process and the potential in the fall-off rates of eigenfunctions is now more subtle. The effect of the potential appears in the relative position of the corresponding eigenvalue from the edge of the continuous spectrum. The effect of the process comes in mainly through two parameter functions expressing (inverse) preference rates for specific jump scenarios. They are defined in (2.12)-(2.13) below and discussed in detail; a third parameter function given by (2.14) plays a technical role only.

Our main results can be roughly summarized as follows.

(1) Whenever $\varphi$ is a positive eigenfunction corresponding to an eigenvalue $\lambda \in \mathbf{R}$, it is bounded from below by the Lévy intensity (Theorem 4.1), i.e.,

$$
\varphi(x) \geq \operatorname{const} \nu(x)
$$

for large enough $|x|$, with a prefactor dependent on the process and the eigenvalue. Moreover, if the basic jump-paring condition (A1.3) below does not hold, then $\varphi$ necessarily decays slower than $\nu$.

(2) When an eigenvalue $\lambda$ is sufficiently low-lying below zero, the corresponding eigenfunction $\varphi$ satisfies

$$
|\varphi(x)| \leq \text { const }\|\varphi\|_{\infty} \nu(x),
$$

for large enough $|x|$, where the constant prefactor depends on the process and the eigenvalue (Theorem 4.2). When $\lambda<0$ is arbitrary, we consider separately symmetric jump-paring processes with Lévy densities that are slowly (see (3.11)) or fast (see (3.16)-(3.17)) decaying at infinity. Under a smallness condition involving the parameter functions, see (2.15)-(2.16) 
and (2.19), we find that the upper bound of $\varphi$ is again driven by the jump intensity as above (Theorem 4.3).

(3) A combination of the upper and lower bounds in (1)-(2) above gives that when an eigenfunction is a ground state, we have

$$
\varphi_{0}(x) \asymp \nu(x) .
$$

This then holds for low-lying bottom eigenvalues $\lambda_{0}$, and any negative $\lambda$ whenever the conditions in (1) are in place. Also, from the above it is seen that (1.3) does apply to the case of decaying potentials in these circumstances. The smallness of the ground state eigenvalue can be understood in terms of the cost of passing a potential barrier plus leaving a ball (Proposition 4.3).

(4) Our present framework offers a unified treatment of both decaying and confining potentials. In Theorem 4.4 we are able to derive an upper bound for eigenfunctions in the case of confining potentials by using the methods developed in this paper, and recover a result in [34] as a bonus.

It is helpful to see to what expressions these results translate in some specific cases. From these behaviours the following interesting phenomenon emerges. For a jump-paring process with Lévy intensity $\nu$ decaying slower than exponentially (e.g., polynomially or sub-exponentially heavytailed) the corresponding ground state has the same fall-off rate as $\nu$ (see Corollaries 4.1-4.2 and Remarks 4.2-4.3). When $\nu$ decays exponentially or faster, the regime qualitatively changes and a "phase transition" in the fall-off rates can be observed. For exponentially decaying Lévy intensity the following dichotomy occurs. If the ground state is an eigenfunction at a sufficiently low-lying eigenvalue, it has the same fall-off as $\nu$, while for bottom eigenvalues which are closer to zero (i.e., to the edge of the continuous spectrum), the fall-off gets much slower, with essential contribution of the eigenvalue into the rate, see Corollary 4.3. (Suggestively, this means that the ground state of such a process in a deep enough potential well decays like $\nu$, decays slower than $\nu$ if the well is not deep enough, and the ground state may even cease to exist if the well is too shallow.) When the process is outside of the jump-paring class, the ground state decays slower than $\nu$ (Theorem 4.1), with significant contribution from the bottom eigenvalue as long as its absolute value is not too large (see Corollaries 4.3-4.4). In particular, the resulting fall-off becomes comparable to that of perturbed Brownian motion (1.1). We note that this phenomenon can be appreciated as another level in the hierarchy of ground state behaviour, and the order of increasingly "regular" properties can be seen as the line evolving from slower than Lévy intensity-driven decay, through Lévy intensity-driven decay or faster, and intrinsic ultracontractivity which is topping this by a uniform ergodic behaviour. For further details see Subsection 4.4 and Remark 4.4, and for comparison with confining potentials we refer to [34, Sects. 2.3-2.5, 4.2].

The mechanism behind these differing behaviours can be heuristically understood as follows. For illustration consider a negative potential well with a single minimum at the origin, tending to zero as $|x| \rightarrow \infty$, with ground state eigenvalue $\lambda_{0}<0$. For energetic reasons the paths will be encouraged to move and spend long times in close neighbourhoods of the bottom of the well (a concentration effect), while for entropic reasons the process tends to explore space arbitrarily far. Furthermore, a lower ground state eigenvalue means a more prominent energetic effect with paths concentrating around the bottom of the well, resembling the case of confining potentials. Stronger concentration means that paths are better localized in space and so the ground state has a faster decay. In this case the fall-off rate is the best possible, and for the processes considered it is given by $\nu$. On the other hand, when $\left|\lambda_{0}\right|$ is not large enough, the energetic effect weakens and the 
efficiency of the concentration mechanism looses out so that the perturbed process behaves more like the free process. However, for a process starting far away from the origin and moving via long direct jumps (which is the case for a jump-paring process) rather than via sequences of long jumps interspersed with smaller fluctuations, this relatively small energetic effect can still be enough for securing an efficient concentration. This again gives a better localization of paths and implies a decay of the ground state determined by $\nu$. The jump property described above is formally expressed by conditions (2.15)-(2.16) and we think of it as the capacity of responsiveness to perturbation of the given process. To complete this picture, we note that for jump-paring processes without this property or for other processes moving in smaller jumps or fluctuating continuously, the paths from far out can cluster around the potential well less efficiently and once they are back around the origin, they spend comparatively large amounts of time building up "backlogs" in the decay-events, so their ground states decay much slower than $\nu$.

In what follows we develop a new methodology to tackle perturbations of jump processes accommodating subtle spectral effects coming from potentials decaying to zero rather than producing a large killing by growing to infinity at infinity. Using a probabilistic representation, the spatial decay of eigenfunctions becomes equivalent to the behaviour of mean hitting times of large balls centered at the origin. The Laplace transforms of such hitting times are harmonic functions with respect to the process killed at a rate given by the absolute value of the corresponding eigenvalue. A first technical novelty in this paper is then a full deployment of a sequence of self-improving estimates on functions which we call harmonic at infinity for the underlying jump-paring Lévy process killed at a non-zero rate (Lemma 3.2). We find that the resulting upper bound driven by $\nu$ holds under the general balancing condition (3.7) involving the killing rate and the pivotal parameter functions (2.12)-(2.14). In Theorem 3.2 we show that this condition is satisfied for Lévy densities with a doubling property for large arguments. Theorem 3.3, which applies to jump-paring processes with light jump intensities, is one of the most involved and crucial technical results in the paper. It states that under the key smallness conditions on the parameter functions (2.15)-(2.16), the Laplace transform of the first hitting time of a closed ball with respect to the path measure of the process starting far from the origin is dominated by $\nu$. In deriving this result we come to a delicate argument based on the domination of $\nu$ by a carefully constructed family of jump intensities to which the fundamental Lemma 3.2 can be applied. We stress that improved estimates in Lemma 3.2 also allow to treat confining potentials now within the same framework, and we are able to recover the decay estimates obtained in [34], see Theorem 4.4 below. In particular, this shows that if one is interested only in decay properties (and not also in intrinsic ultracontractivity), a detailed tracking of the jumps as done in the cited paper is not needed, and we obtain a unified and streamlined treatment. We note that all upper estimates obtained in the present paper are sharp in the sense that they are directly governed by $\nu(x)$ rather than $\nu(c x)$ with some $c \in(0,1)$, even when $\nu$ is very light at infinity.

The remainder of this paper is organized as follows. In Section 2 we introduce and discuss the class of underlying Lévy processes, the corresponding parameter functions, potentials and Feynman-Kac semigroups considered in this paper. Section 3 is devoted to proving the technical estimates for functions harmonic at infinity. In Section 4 we come to present the fall-off properties of eigenfunctions, including a detailed discussion of decay rates for specific classes of processes and operators. 


\section{Jump-paring Lévy processes and non-local Schrödinger operators}

\subsection{Class of Lévy processes}

In this subsection we introduce the class of Lévy processes considered in this paper and discuss some of their properties which will be used below.

Let $\left(X_{t}\right)_{t \geq 0}$ be a symmetric Lévy process with values in $\mathbf{R}^{d}, d \geq 1$, with probability measure $\mathbf{P}^{x}$ of the process starting from $x \in \mathbf{R}^{d}$. We use the notation $\mathbf{E}^{x}$ for expectation with respect to $\mathbf{P}^{x}$. Recall that $\left(X_{t}\right)_{t \geq 0}$ is a Markov process with respect to its natural filtration, satisfying the strong Markov property and having càdlàg paths. It is determined by the characteristic function

$$
\mathbf{E}^{0}\left[e^{i \xi \cdot X_{t}}\right]=e^{-t \psi(\xi)}, \quad \xi \in \mathbf{R}^{d}, t>0
$$

with the characteristic exponent given by the Lévy-Khintchin formula

$$
\psi(\xi)=A \xi \cdot \xi+\int_{\mathbf{R}^{d}}(1-\cos (\xi \cdot z)) \nu(d z)
$$

Here $A=\left(a_{i j}\right)_{1 \leq i, j \leq d}$ is a symmetric non-negative definite matrix, and $\nu$ is a symmetric Lévy measure on $\mathbf{R}^{d} \backslash\{0\}$, i.e., $\int_{\mathbf{R}^{d}}\left(1 \wedge|z|^{2}\right) \nu(d z)<\infty$ and $\nu(E)=\nu(-E)$, for every Borel set $E \subset$ $\mathbf{R}^{d} \backslash\{0\}$, thus the Lévy triplet of the process is $(0, A, \nu)$.

In the present paper we assume throughout that the Lévy measure appearing in (2.1) is an infinite measure and it is absolutely continuous with respect to Lebesgue measure, i.e.,

$$
\nu\left(\mathbf{R}^{d} \backslash\{0\}\right)=\infty \quad \text { and } \quad \nu(d x)=\nu(x) d x, \quad \text { with } \quad \nu(x)>0 .
$$

For simplicity, we denote the density of the Lévy measure also by $\nu$ as it is the object we will use below. When $A \equiv 0$, the random process $\left(X_{t}\right)_{t \geq 0}$ is said to be a purely jump process. Note that the properties (2.2) jointly imply that $\left(X_{t}\right)_{t \geq 0}$ is a strong Feller process, or equivalently, its onedimensional distributions are absolutely continuous with respect to Lebesgue measure, i.e., there exist measurable transition densities $p(t, x, y)=p(t, 0, y-x)=: p(t, y-x)$ such that $\mathbf{P}^{0}\left(X_{t} \in E\right)=$ $\int_{E} p(t, x) d x$, for every Borel set $E \subset \mathbf{R}^{d}$ (see e.g. [46, Th. 27.7]).

We will make use below of the following symmetrization of the exponent $\psi$. Denote

$$
\Psi(r)=\sup _{|\xi| \leq r} \psi(\xi), \quad r>0 .
$$

It follows from a combination of [48, Rem. 4.8] and [44, Sect. 3] (see also direct calculations with explicit constants in [24, Lem. 4]) that there exist $C_{1}, C_{2}>0$, independent of the process (i.e., of $A$ and $\nu)$, such that

$$
C_{1} H\left(\frac{1}{r}\right) \leq \Psi(r) \leq C_{2} H\left(\frac{1}{r}\right), r>0, \quad \text { where } \quad H(r)=\frac{\|A\|}{r^{2}}+\int_{\mathbf{R}^{d}}\left(1 \wedge \frac{|y|^{2}}{r^{2}}\right) \nu(d y) .
$$

It can be directly checked that $H$ is non-increasing and the doubling property $H(r) \leq 4 H(2 r)$, $r>0$, holds. In particular, it follows that $\Psi(2 r) \leq 4 C_{1}^{-1} C_{2} \Psi(r)$, for all $r>0$.

The generator $L$ of the process $\left(X_{t}\right)_{t \geq 0}$ is uniquely determined by its Fourier symbol

$$
\widehat{L f}(\xi)=-\psi(\xi) \widehat{f}(\xi), \quad \xi \in \mathbf{R}^{d}, f \in D(L),
$$


with domain $D(L)=\left\{f \in L^{2}\left(\mathbf{R}^{d}\right): \psi \widehat{f} \in L^{2}\left(\mathbf{R}^{d}\right)\right\}$. It is a negative non-local self-adjoint operator with core $C_{0}^{\infty}\left(\mathbf{R}^{d}\right)$, and

$$
L f(x)=\sum_{i, j=1}^{d} a_{i j} \frac{\partial^{2} f}{\partial x_{j} \partial x_{i}}(x)+\lim _{\varepsilon \searrow 0} \int_{|y-x|>\varepsilon}(f(y)-f(x)) \nu(y-x) d y, \quad x \in \mathbf{R}^{d}, f \in C_{0}^{\infty}\left(\mathbf{R}^{d}\right) .
$$

The corresponding Dirichlet form $(\mathcal{E}, D(\mathcal{E}))$ is defined by

$$
\mathcal{E}(f, g)=\int_{\mathbf{R}^{d}} \psi(\xi) \widehat{f}(\xi) \overline{\widehat{g}(\xi)} d \xi, \quad f, g \in D(\mathcal{E}),
$$

where $D(\mathcal{E})=\left\{f \in L^{2}\left(\mathbf{R}^{d}\right): \int_{\mathbf{R}^{d}} \psi(\xi)|\widehat{f}(\xi)|^{2} d \xi<\infty\right\}$. If $f \in D(L)$, then $\mathcal{E}(f, g)=(-L f, g)$. Furthermore, let

$$
\mathcal{B}=\left\{f \in C_{\mathrm{c}}^{2}\left(\mathbf{R}^{d}\right): f(x)=1 \text { for } x \in B(0,1 / 2), f(x)=0 \text { for } x \in B(0,1)^{c} \text { and } 0 \leq f \leq 1\right\},
$$

and notice that for $f_{s}(x)=f(x / s)$ with $f \in \mathcal{B}$ and $s>0$, we have

$$
\begin{aligned}
\left\|L f_{s}\right\|_{\infty} & \leq 2 \nu\left(B(0, s)^{c}\right)+\frac{1}{s^{2}} \sup _{i, j=1, \ldots, d}\left\|\frac{\partial^{2} f}{\partial x_{i} \partial x_{j}}\right\|_{\infty}\left(\|A\|+\int_{|y| \leq s}|y|^{2} \nu(d y)\right) \\
& \leq\left(2 \vee \sup _{i, j=1, \ldots, d}\left\|\frac{\partial^{2} f}{\partial x_{i} \partial x_{j}}\right\|_{\infty}\right) H(s), \quad s>0 .
\end{aligned}
$$

Denote

$$
C_{3}(X, s):=\inf _{f \in \mathcal{B}}\left\|L f_{s}\right\|_{\infty}, \quad \text { with } \quad f_{s}(x)=f(x / s), \quad s>0 .
$$

By the above we have

$$
C_{3}(X, s) \leq C_{1}^{-1}\left(2 \vee \inf _{f \in \mathcal{B}} \sup _{i, j=1, \ldots, d}\left\|\frac{\partial^{2} f}{\partial x_{i} \partial x_{j}}\right\|_{\infty}\right) \Psi(1 / s), \quad s>0 .
$$

For more details on Lévy processes we refer to $[5,3,46,30]$.

Let $D \subset \mathbf{R}^{d}$ be an open bounded set and consider the first exit time $\tau_{D}=\inf \left\{t \geq 0: X_{t} \notin D\right\}$ from $D$. The transition densities $p_{D}(t, x, y)$ of the process killed upon exiting $D$ are given by the Dynkin-Hunt formula

$$
p_{D}(t, x, y)=p(t, y-x)-\mathbf{E}^{x}\left[\tau_{D}<t ; p\left(t-\tau_{D}, y-X_{\tau_{D}}\right)\right], \quad x, y \in D .
$$

The Green function of the process $\left(X_{t}\right)_{t \geq 0}$ on $D$ is thus $G_{D}(x, y)=\int_{0}^{\infty} p_{D}(t, x, y) d t$, for all $x, y \in D$. Furthermore, by [48, Rem. 4.8] we have

$$
\mathbf{E}^{0}\left[\tau_{B(0, r)}\right] \leq \frac{C_{4}}{\Psi(1 / r)}, \quad r>0
$$

with a constant $C_{4}$ independent of the process.

We will use throughout the notation $C(a, b, c, \ldots)$ for a positive constant dependent on parameters $a, b, c, \ldots$, while dependence on the process $X:=\left(X_{t}\right)_{t \geq 0}$ is indicated by $C(X)$, and dependence on the dimension $d$ is assumed without being stated explicitly. Since constants appearing in definitions, lemmas and theorems play an important role in this paper, we use the numbering $C_{1}, C_{2}, \ldots$ to be able to track them. We will also use the notation $f \asymp C g$ meaning that $C^{-1} g \leq f \leq C g$ with a constant $C \geq 1$, while $f \asymp g$ means that there is a constant $C \geq 1$ such that the latter holds. By $f \approx g$ we understand that $\lim _{|x| \rightarrow \infty} f(x) / g(x)=1$. In proofs $c_{1}, c_{2}, \ldots$ will be used to denote auxiliary constants. 
We will use the following class of Lévy processes.

Definition 2.1 (Symmetric jump-paring Lévy processes). Let $\left(X_{t}\right)_{t \geq 0}$ be a Lévy process with Lévy-Khintchin exponent $\psi$ as in (2.1)-(2.2) and Lévy triplet $(0, A, \nu)$, satisfying the following conditions.

(A1) Lévy intensity: There exist a profile function $g:(0, \infty) \rightarrow(0, \infty)$ and a constant $C_{5}=$ $C_{5}(X)$ such that

$$
\nu(x) \asymp C_{5} g(|x|), \quad x \in \mathbf{R}^{d} \backslash\{0\},
$$

and the following properties hold:

(A1.1) $g$ is non-increasing on $(0, \infty)$

(A1.2) there exists a constant $C_{6}=C_{6}(X)$ such that

$$
g(|x|) \leq C_{6} g(|x|+1), \quad|x| \geq 1
$$

(A1.3) there exists a constant $C_{7}=C_{7}(X)$ such that

$$
\int_{\substack{|x-y|>1 \\|y|>1}} g(|x-y|) g(|y|) d y \leq C_{7} g(|x|), \quad|x| \geq 1 .
$$

(A2) Transition density: There exists $t_{\mathrm{b}}>0$ such that $\sup _{x \in \mathbf{R}^{d}} p\left(t_{\mathrm{b}}, x\right)=p\left(t_{\mathrm{b}}, 0\right)<\infty$.

(A3) Green function: For all $0<p<q<R<\infty$ we have

$$
\sup _{x \in B(0, p)} \sup _{y \in B(0, q)^{c}} G_{B(0, R)}(x, y)<\infty .
$$

We call $\left(X_{t}\right)_{t \geq 0}$ satisfying the above conditions a symmetric jump-paring Lévy process and refer to the convolution condition in (A1.3) as the jump-paring property.

Assumptions (A1.1)-(A1.2) are self-explanatory. It can be directly shown that these conditions and a similar geometric argument as in [34, Lem. 3.4(2)]) imply

$$
\int_{\substack{r<|z| \leq r+1 \\|y-z|>1 / 8}} \nu(z-y) d z \leq C_{8} \int_{r-1<|z| \leq r} \nu(z-y) d z, \quad|y| \geq r+1, r \geq 1,
$$

with a constant $C_{8}=C_{8}(X) \geq 1$, independent of $r$. The bound in (A1.3) provides a control of the convolutions of $\nu$ with respect to large jumps and has a structural importance in defining the class of processes we consider. It says that the intensity of double large jumps of the process are dominated by the intensity of a single large jump. Let $\nu_{1}(x)=\nu(x) \mathbf{1}_{B(0,1)^{c}}(x)$. It is then seen iteratively that under (A1.3) in fact

$$
\nu_{1}^{n *}(x) \leq C^{n-1} \nu_{1}(x), \quad|x| \geq 1, n \in \mathbf{N},
$$

holds, which means that every sequence of any finite length of large jumps of the process is dominated by single large jumps, which gives the name to the class of Lévy processes above.

The convolution condition (A1.3) has been introduced in [34] and proved to be a strong tool in studying large-scale properties of jump Lévy processes. Recently, in [37] it was also used to characterize the short-time behaviour of heat kernels for a large class of convolution semigroups. It can be easily checked that (A1.3) in fact implies (A1.2), see [37, Lem. 1(a)], however, for completeness and more clarity we prefer to state (A1.2) in Definition 2.1 separately.

Assumption (A2) is equivalent with $e^{-t_{\mathrm{b}} \psi} \in L^{1}\left(\mathbf{R}^{d}\right)$, for some $t_{\mathrm{b}}>0$. In this case $p\left(t_{\mathrm{b}}, x\right)$ can be obtained by the Fourier inversion formula. Clearly, this property extends to all $t \geq t_{\mathrm{b}}$ by the Markov property of $\left(X_{t}\right)_{t \geq 0}$. For more details on the existence and properties of transition probability densities for Lévy processes we refer to [40] and references therein. Finally, we remark 
that in many cases of interest Assumption (A3) follows directly from (rough) space-time estimates of the densities $p(t, x)$. Indeed, if Assumption (A2) holds and for every $r>0$ there exists $C=C(r)$ such that $\sup _{|x| \geq r} p(t, x) \leq C t, t>0$, then (A3) follows by a standard estimate as in (2.22) below.

The range of processes satisfying Assumptions (A1)-(A3) is wide including large subclasses of isotropic unimodal Lévy processes, subordinate Brownian motions, Lévy processes with nondegenerate Brownian components, symmetric stable-like processes, or processes with subexponentially or exponentially localized Lévy measures. In particular, it covers all the examples discussed in detail in [34, Sect. 4].

\subsection{Parameter functions and jump scenarios}

Next we introduce some functions of the Lévy processes considered through which the decay of eigenfunctions of the non-local Schrödinger operators will be analyzed. As it will be seen, they relate to some special features of the process, which to our knowledge have not been addressed in the literature before.

(1) Define

$$
K_{1}^{X}(s):=\sup _{|x| \geq s} \frac{\int_{|x-y|>s,|y|>s} \nu(x-y) \nu(y) d y}{\nu(x)}, \quad s \geq 1 .
$$

Notice that from $(\mathrm{A} 1.3)$ it follows that $K_{1}^{X}:[1, \infty) \rightarrow\left(0, C_{5}^{3} C_{7}\right]$ is a non-increasing function and gives the optimal constant $C$ in the bound

$$
\int_{\substack{|x-y|>s \\|y|>s}} \nu(x-y) \nu(y) d y \leq C \nu(x), \quad|x| \geq s,
$$

for any fixed $s \geq 1$. Thus $1 / K_{1}^{X}(s)$ is the rate of preference of single jumps of size at least $s$ over double jumps of size at least $s$ each, i.e., when $K_{1}^{X}(s)$ decreases with $s \rightarrow \infty$, this preference improves.

(2) Let $0<s_{1}<s_{2}<s_{3} \leq \infty$ and define

$$
K_{2}^{X}\left(s_{1}, s_{2}, s_{3}\right):=\inf \left\{C \geq 1: \nu(x-y) \leq C \nu(x),|y| \leq s_{1}, s_{2} \leq|x|<s_{3}\right\} .
$$

Using (A1.1)-(A1.2) we see that $K_{2}^{X}\left(s_{1}, s_{2}, s_{3}\right)$ is well-defined and a non-decreasing function in $s_{1} \in\left(0, s_{2}\right)$, for every fixed $0<s_{2}<s_{3}$. Whenever $s_{3}=\infty$ and $s_{2} \gg s_{1} \gg 1$, which will be mostly the case considered below, $1 / K_{2}^{X}\left(s_{1}, s_{2}, s_{3}\right)$ can be interpreted to measure the rate of preference of a direct large jump from $x \in B\left(0, s_{2}\right)^{c}$ to 0 over a jump from a point $z$ situated in the $s_{1}$-neighbourhood of $x$ to 0 . In our applications, the second case corresponds to the situation when the process moving from $x$ to 0 first fluctuates inside $B\left(x, s_{1}\right)$ and then makes one large jump to the origin. On this account, we refer to $K_{2}^{X}$ as the inverse rate of preference of the scenario "no small steps but direct large jump" over the scenario "first small steps, then large jump".

(3) Let assumption (A3) hold and define $K_{3}^{X}:(0, \infty) \rightarrow(0, \infty)$ by

$$
K_{3}^{X}(s):=\sup _{x, y:|x-y| \geq s / 8} G_{B(0, s)}(x, y), \quad s>0 .
$$

These parameters will be relevant through their behaviour in some jump scenarios which we discuss next. First consider the following asymptotic properties involving $K_{1}^{X}$ and $K_{2}^{X}$ :

$$
\text { there exists } \kappa_{1} \geq 2 \text { such that } \lim _{s \rightarrow \infty} K_{1}^{X}\left(\kappa_{1} s\right) K_{2}^{X}\left(s, \kappa_{1} s, \infty\right)=0
$$


and

(2.16) there exists $\kappa_{2}<\infty$ such that for all $s_{1} \geq 1$ we have $\limsup _{s \rightarrow \infty} K_{2}^{X}\left(s_{1}, s, \infty\right) \leq \kappa_{2}$.

Due to the roles played by conditions (2.15)-(2.16) in Sections 3-4 below, we think of them as expressing the capacity of responsiveness to perturbation of the process $\left(X_{t}\right)_{t \geq 0}$. Note that

$$
\lim _{s \rightarrow \infty} K_{1}^{X}(s)=0
$$

is necessary but not sufficient for (2.15), even if (2.16) holds.

To understand what (2.15) means, first notice that by the definition of $K_{1}^{X}$ it follows that

$$
\int_{\substack{|x-z|>s_{2} \\|z|>s_{2}}} \nu(x-z) \nu(z) d z \leq K_{1}^{X}\left(s_{2}\right) \nu(x), \quad \text { for all } x \text { such that }|x|>s_{2},
$$

and using the definition of $K_{2}^{X}$ we have

$$
\nu(x) \leq K_{2}^{X}\left(s_{1}, s_{2}, \infty\right) \nu(w), \quad \text { for all } w \text { such that }|w|>s_{2},|x-w|<s_{1} .
$$

Thus $1 /\left(K_{1}^{X}\left(s_{2}\right) K_{2}^{X}\left(s_{1}, s_{2}, \infty\right)\right)$ measures the rate of preference of a single large jump from $w$ to the origin ("direct single large jump" scenario) over two large jumps from $x \in B\left(w, s_{1}\right)$ to 0 ("first small steps, then two large jumps" scenario). This means that property (2.15) corresponds to the situation when the first scenario outdoes the second at a rate which improves when the length of the long jumps increases appropriately with the scale of the smaller fluctuations. Secondly, observe that (2.16) says that the "no small steps but direct large jump" scenario above outdoes the "first small steps, then large jump" scenario at a rate $1 / \kappa_{2}$, no matter how large the smaller fluctuations are (i.e., there is always a suitably larger jump for which the former is preferred to occur).

Next we show that $K_{1}^{X}$ dominates the tail of the corresponding Lévy measure.

Lemma 2.1. Under Assumption (A1) we have

$$
K_{1}^{X}(s) \geq \frac{\nu\left(B(0, s)^{c}\right)}{2 C_{5}^{4}}, \quad s \geq 1
$$

Proof. Let $s \geq 1$ and denote $x_{s, n}=(2 s+n, 0, \ldots, 0), n \in \mathbf{N}$. By the definition of $K_{1}^{X}$ and the monotonicity of $g$, for every $n \in \mathbf{N}$ we have

$$
\begin{aligned}
C_{5} K_{1}^{X}(s) g(2 s+n) & \geq K_{1}^{X}(s) \nu\left(x_{s, n}\right) \geq \int_{\substack{\left|x_{s, n-y \mid>s}\right| y|>s\\
| y \mid>}} \nu\left(x_{s, n}-y\right) \nu(y) d y \\
& \geq \frac{1}{C_{5}^{2}} \int_{\substack{\left|x_{s, n}-y\right|>s \\
|y|>s}} g\left(\left|x_{s, n}-y\right|\right) g(|y|) d y \geq \frac{g(2 s+n)}{C_{5}^{2}} \int_{\substack{s<\left|x_{s, n}-y\right|<2 s+n \\
s<|y|<s+n}} g(|y|) d y .
\end{aligned}
$$

For every $y=\left(y_{1}, \ldots, y_{d}\right)$ such that $y_{1}>0$ we have $\left|y-x_{s, n}\right|<2 s+n$ for sufficiently large $n \in \mathbf{N}$. Hence, for every $y \in \mathbf{R}^{d}$,

$$
\mathbf{1}_{\left\{z: s<\left|x_{s, n}-z\right|<2 s+n, s<|z|<s+n\right\}}(y) \rightarrow \mathbf{1}_{\left\{z:|z|>s, z_{1}>0\right\}}(y) \quad \text { as } n \rightarrow \infty .
$$

Using Fatou's lemma and radial symmetry, this implies

$$
C_{5}^{3} K_{1}^{X}(s) \geq \liminf _{n \rightarrow \infty} \int_{\substack{s<\left|x_{s, n}-y\right|<2 s+n \\ s<|y|<s+n}} g(|y|) d y \geq \int_{|y|>s, y_{1}>0} g(|y|) d y=\frac{1}{2} \int_{|y|>s} g(|y|) d y .
$$

Applying (A1) again, we obtain the claimed bound. 
Finally, consider $K_{3}^{X}$. We will require it to satisfy

$$
\sup _{s \geq 1}\left[K_{3}^{X}(s) \Psi(1 / s) s^{d}\right]<\infty .
$$

This is a regularity condition known to hold for a large class of processes, in particular, for isotropic unimodal Lévy processes (i.e., $A \equiv a \operatorname{Id}$ for some $a \geq 0$ and $\nu(x)$ is a non-increasing radial function) and $d \geq 3$, see [24, Th. 3]. It is reasonable to conjecture that actually (2.19) is valid in a generality which covers all the cases considered in the present paper, but a general argument does not seem to be available. While this condition is not decisive for our results below, we use it as a convenient technical assumption. The following lemma gives a sufficient condition.

For a continuous non-decreasing function $\Phi:[0, \infty) \rightarrow[0, \infty)$ such that $\Phi(0)=0$ and $\lim _{r \rightarrow \infty} \Phi(r)=$ $\infty$ we denote

$$
\Phi^{-1}(s)=\sup \{r \geq 0: \Phi(r)=s\} \quad \text { and } \quad \Phi_{*}^{-1}(s)=\inf \{r \geq 0: \Phi(r)=s\}, \quad s \geq 0,
$$

so that $\Phi\left(\Phi^{-1}(s)\right)=\Phi\left(\Phi_{*}^{-1}(s)\right)=s, \Phi^{-1}(\Phi(s)) \geq s$ and $\Phi_{*}^{-1}(\Phi(s)) \leq s$ for $s \geq 0$.

Lemma 2.2. Let $\left(X_{t}\right)_{t \geq 0}$ be a Lévy process determined by the Lévy-Khintchin exponent $\psi$ as in (2.1) such that (2.2) holds. Suppose, moreover, that there exists a continuous non-decreasing function $\Phi:[0, \infty) \rightarrow[0, \infty)$ such that $\Phi(0)=0$ and $\lim _{r \rightarrow \infty} \Phi(r)=\infty$, with the doubling property $\Phi(2 s) \leq$ $C \Phi(s), s>0$, and a number $\Theta \geq 0$ for which

$$
\sup _{|x| \geq r} p(t, x) \leq C_{9} t\left(\frac{\Phi(1 / r)}{r^{d}}+\Theta\right), \quad t>0, \quad r \geq 1,
$$

and

$$
p(t, 0)=\int_{\mathbf{R}^{d}} e^{-t \psi(z)} d z \leq C_{10}\left(\Phi_{*}^{-1}\left(\frac{1}{t}\right)\right)^{d}, \quad t \geq t_{0},
$$

for some $t_{0}>0$ and constants $C_{9}, C_{10}$. Then there exists $r_{0} \geq 1$ such that

$$
K_{3}^{X}(r) \leq 8^{d} e C^{3} C_{9} \frac{1}{\Phi(1 / r) r^{d}}+e C_{9} \frac{\Theta}{\Phi(1 / r)^{2}}+4 C_{1} C_{2} C_{4} C_{10} \frac{1}{\Psi(1 / r) r^{d}}, \quad r \geq r_{0} .
$$

In particular, if this is true with $\Phi=\Psi$ and $\Theta=0$, then (2.19) holds.

Proof. Starting from the general estimate [10, Prop. 2.3], for $t \geq t_{0}$ and $\eta>0$ we have

$$
\sup _{\{(x, y):|x-y| \geq r / 8\}} G_{B(0, r)}(x, y) \leq e^{\eta t} \sup _{|z| \geq r / 8} \int_{0}^{\infty} e^{-\eta s} p(s, z) d s+\mathbf{E}^{0}\left[\tau_{B(0,2 r)}\right] \int_{\mathbf{R}^{d}} e^{-t \psi(z)} d z .
$$

By (2.20) it follows that

$$
\sup _{|z| \geq r / 8} \int_{0}^{\infty} e^{-\eta s} p(s, z) d s \leq C_{9}\left(8^{d} \frac{\Phi(8 / r)}{\eta^{2} r^{d}}+\frac{\Theta}{\eta^{2}}\right), \quad r \geq 1 .
$$

Taking $\eta=1 / t$ with $t=1 / \Phi(1 / r)$ in $(2.22)$, and using $(2.21),(2.10)$ and the doubling property of $\Phi$, we obtain the claimed inequality.

Below we will mainly consider condition (2.20) in the form

$$
\sup _{|x| \geq r} p(t, x) \leq C_{9} \frac{t \Psi(1 / r)}{r^{d}}, \quad t>0, \quad r \geq 1,
$$

i.e., when $\Phi=\Psi$ and $\Theta=0$. Moreover, we will often use the property that if $\Psi(r) \asymp r^{\alpha}, r \in\left[0, r_{0}\right]$, for some $\alpha>0$ and $r_{0}>0$, then $\Psi^{-1}(r) \asymp \Psi_{*}^{-1}(r) \asymp r^{1 / \alpha}, r \in\left[0, \Psi\left(r_{0}\right)\right]$. Conditions $(2.20)-(2.21)$ and (2.23) will be discussed and illustrated on some specific cases in the next sections. 


\subsection{Feynman-Kac semigroup and non-local Schrödinger operator}

We now give the class of potentials which will be used in this paper.

Definition 2.2 ( $X$-Kato class). We say that the Borel function $V: \mathbf{R}^{d} \rightarrow \mathbf{R}$ called potential belongs to Kato-class $\mathcal{K}^{X}$ associated with the Lévy process $\left(X_{t}\right)_{t>0}$ if it satisfies

$$
\lim _{t \downarrow 0} \sup _{x \in \mathbf{R}^{d}} \mathbf{E}^{x}\left[\int_{0}^{t}\left|V\left(X_{s}\right)\right| d s\right]=0 .
$$

Also, we say that $V$ is an $X$-Kato decomposable potential, denoted $V \in \mathcal{K}_{ \pm}^{X}$, whenever

$$
V=V_{+}-V_{-}, \quad \text { with } \quad V_{-} \in \mathcal{K}^{X} \quad \text { and } \quad V_{+} \in \mathcal{K}_{\text {loc }}^{X},
$$

where $V_{+}, V_{-}$denote the positive and negative parts of $V$, respectively, and where $V_{+} \in \mathcal{K}_{\text {loc }}^{X}$ means that $V_{+} 1_{B} \in \mathcal{K}^{X}$ for all compact sets $B \subset \mathbf{R}^{d}$.

For simplicity, in what follows we refer to $X$-Kato decomposable potentials as $X$-Kato class potentials. It is straightforward to see that $L_{\text {loc }}^{\infty}\left(\mathbf{R}^{d}\right) \subset \mathcal{K}_{\text {loc }}^{X}$. Moreover, by stochastic continuity of $\left(X_{t}\right)_{t \geq 0}$ also $\mathcal{K}_{\text {loc }}^{X} \subset L_{\text {loc }}^{1}\left(\mathbf{R}^{d}\right)$, and thus an $X$-Kato class potential is locally absolutely integrable. Note that condition (2.24) allows local singularities of $V$. For specific processes $\left(X_{t}\right)_{t \geq 0}$ the definition of $X$-Kato class can be explicitly reformulated in an analytic way in terms of the kernel $p(t, x)$ restricted to small $t$ and small $x$. It is shown in [25, Cor. 1.3] that (2.24) is equivalent with

$$
\lim _{t \rightarrow 0^{+}} \sup _{x \in \mathbf{R}^{d}} \int_{0}^{t} \int_{B(x, t)} p(s, x-y)|V(y)| d y d s=0 .
$$

Define

$$
T_{t} f(x)=\mathbf{E}^{x}\left[e^{-\int_{0}^{t} V\left(X_{s}\right) d s} f\left(X_{t}\right)\right], \quad f \in L^{2}\left(\mathbf{R}^{d}\right), t>0 .
$$

By standard arguments based on Khasminskii's Lemma, see [16, Cor.Prop.3.8],[42, Lem.3.37-3.38], for an $X$-Kato class potential $V$ it follows that there exist constants $C_{11}=C_{11}(X, V)$ and $C_{12}=$ $C_{12}(X, V)$ such that

$$
\sup _{x \in \mathbf{R}^{d}} \mathbf{E}^{x}\left[e^{-\int_{0}^{t} V\left(X_{s}\right) d s}\right] \leq \sup _{x \in \mathbf{R}^{d}} \mathbf{E}^{x}\left[e^{\int_{0}^{t} V_{-}\left(X_{s}\right) d s}\right] \leq C_{11} e^{C_{12} t}, \quad t>0 .
$$

Using the Markov property and stochastic continuity of the process it can be shown that $\left\{T_{t}: t \geq 0\right\}$ is a strongly continuous semigroup of symmetric operators on $L^{2}\left(\mathbf{R}^{d}\right)$, which we call the FeynmanKac semigroup associated with the process $\left(X_{t}\right)_{t \geq 0}$ and potential $V$. In particular, by the HilleYoshida theorem there exists a self-adjoint operator $H$, bounded from below, such that $e^{-t H}=T_{t}$. We call the operator $H$ a non-local Schrödinger operator based on the infinitesimal generator $L$ of the process $\left(X_{t}\right)_{t \geq 0}$. Since any $X$-Kato class potential is relatively form bounded with respect to the "free Hamiltonian" $H_{0}=-L$ with relative bound less than 1 , we have $H=H_{0}+V$, where the latter operator is defined in form sense [19, Ch. 2]. For subordinate Brownian motions, $H$ becomes a non-local Schrödinger operator with a Bernstein function of the Laplacian studied in [27], i.e., has the form $\phi(-\Delta)+V$, where $\phi$ is the Laplace exponent of the corresponding subordinator.

We now summarize the basic properties of the operators $T_{t}$ which will be useful below.

Lemma 2.3. Let $\left(X_{t}\right)_{t \geq 0}$ be a symmetric Lévy process with Lévy-Khintchin exponent satisfying (2.1)-(2.2) such that Assumption (A2) holds, and let $V$ be an $X$-Kato class potential. Then the following properties hold: 
(1) For all $t>0$, every $T_{t}$ is a bounded operator on every $L^{p}\left(\mathbf{R}^{d}\right)$ space, $1 \leq p \leq \infty$. The operators $T_{t}: L^{p}\left(\mathbf{R}^{d}\right) \rightarrow L^{p}\left(\mathbf{R}^{d}\right)$ for $1 \leq p \leq \infty, t>0$, and $T_{t}: L^{p}\left(\mathbf{R}^{d}\right) \rightarrow L^{\infty}\left(\mathbf{R}^{d}\right)$ for $1<p \leq \infty, t \geq t_{\mathrm{b}}$, and $T_{t}: L^{1}\left(\mathbf{R}^{d}\right) \rightarrow L^{\infty}\left(\mathbf{R}^{d}\right)$ for $t \geq 2 t_{\mathrm{b}}$ are bounded, with some $t_{\mathrm{b}}>0$.

(2) For all $t \geq 2 t_{\mathrm{b}}, T_{t}$ has a bounded measurable kernel $u(t, x, y)$ symmetric in $x$ and $y$, i.e., $T_{t} f(x)=\int_{\mathbf{R}^{d}} u(t, x, y) f(y) d y$, for all $f \in L^{p}\left(\mathbf{R}^{d}\right)$ and $1 \leq p \leq \infty$.

(3) For all $t>0$ and $f \in L^{\infty}\left(\mathbf{R}^{d}\right), T_{t} f$ is a bounded continuous function.

(4) For all $t \geq 2 t_{\mathrm{b}}$ the operators $T_{t}$ are positivity improving, i.e., $T_{t} f(x)>0$ for all $x \in \mathbf{R}^{d}$ and $f \in L^{2}\left(\mathbf{R}^{d}\right)$ such that $f \geq 0$ and $f \neq 0$ a.e.

The above properties can be established by standard arguments, see [16, Sect. 3.2]. Note that we do not assume that $p(t, x)$ is bounded for all $t>0$, and thus in general the operators $T_{t}: L^{p}\left(\mathbf{R}^{d}\right) \rightarrow$ $L^{\infty}\left(\mathbf{R}^{d}\right)$ need not be bounded for $t<t_{\mathrm{b}}$.

Related to the Feynman-Kac semigroup, we also define the potential operator by

$$
G^{V} f(x)=\int_{0}^{\infty} T_{t} f(x) d t=\mathbf{E}^{x}\left[\int_{0}^{\infty} e^{-\int_{0}^{t} V\left(X_{s}\right) d s} f\left(X_{t}\right) d t\right]
$$

for non-negative or bounded Borel functions $f$ on $\mathbf{R}^{d}$. Recall that $\tau_{D}$ denotes the first exit time of the process from domain $D$. Whenever $D \subset \mathbf{R}^{d}$ is an open set and $f$ is a non-negative or bounded Borel function on $\mathbf{R}^{d}$, it follows by the strong Markov property of the process that for every $x \in D$

$$
G^{V} f(x)=\mathbf{E}^{x}\left[\int_{0}^{\tau_{D}} e^{-\int_{0}^{t} V\left(X_{s}\right) d s} f\left(X_{t}\right) d t\right]+\mathbf{E}^{x}\left[\tau_{D}<\infty ; e^{-\int_{0}^{\tau_{D}} V\left(X_{s}\right) d s} G^{V} f\left(X_{\tau_{D}}\right)\right] .
$$

For background on potential theory we refer to $[16,6,5,7,15,9,10]$.

\section{Estimates of harmonic functions}

\subsection{Lower bound for functions harmonic at infinity}

Using (2.27) it will be seen that eigenfunctions for a given process and a given potential are comparable to specific harmonic functions; this will be explored to a large extent by deriving and using the representation (4.5) below. In this section first we develop some technical tools concerning harmonic functions.

Let $\eta>0$. Recall that a non-negative Borel function $f$ on $\mathbf{R}^{d}$ is called $(X, \eta)$-harmonic in an open set $D \subset \mathbf{R}^{d}$ if

$$
f(x)=\mathbf{E}^{x}\left[\tau_{U}<\infty ; e^{-\eta \tau_{U}} f\left(X_{\tau_{U}}\right)\right], \quad x \in U,
$$

for every open set $U$ with its closure $\bar{U}$ contained in $D$, and it is called regular $(X, \eta)$-harmonic in $D$ if (3.1) holds for $U=D$ (where $\tau_{U}$ is the first exit time from $U$ ). By the strong Markov property every regular $(X, \eta)$-harmonic function in $D$ is $(X, \eta)$-harmonic in $D$. Below we mainly consider the case when (3.1) holds with $D=\bar{B}(0, r)^{c}$ for some $r>0$. We refer to this property as harmonicity at infinity.

We also recall that when $D \subset \mathbf{R}^{d}$ is a bounded open domain, the following formula due to Ikeda and Watanabe holds [29, Th. 1]: for every $\eta>0$ and every bounded or non-negative Borel function $f$ on $\mathbf{R}^{d}$ such that $\operatorname{dist}(\operatorname{supp} f, D)>0$, we have

$$
\mathbf{E}^{x}\left[e^{-\eta \tau_{D}} f\left(X_{\tau_{D}}\right)\right]=\int_{D} \int_{0}^{\infty} e^{-\eta t} p_{D}(t, x, y) d t \int_{D^{c}} f(z) \nu(z-y) d z d y, \quad x \in D .
$$


The next theorem is our first result in this section. It states that non-negative functions that are $(X, \eta)$-harmonic at infinity are bounded from below by $\nu$, no matter how small $\eta$ is. On the other hand, it says that when condition (A1.2), or at least the jump-paring property (A1.3), fails to hold, then such functions will not be dominated by $\nu$ at infinity even for large $\eta$. This means that it is reasonable to ask which properties of jump-paring Lévy processes will guarantee that the functions $(X, \eta)$-harmonic at infinity are comparable to $\nu$ at least far away from the origin.

Theorem 3.1. Let $\eta>0$ and $\left(X_{t}\right)_{t \geq 0}$ be a symmetric Lévy process with Lévy-Khintchin exponent satisfying (2.1)-(2.2). Let $r>0$ and $f$ be a non-negative $(X, \eta)$-harmonic function in $\bar{B}(0, r)^{c}$ such that $\int_{B(0, r)} f(z) d z>0$. Then we have the following:

(1) If (A1.1)-(A1.2) hold, then

$$
f(x) \geq\left(\frac{1-e^{-\eta}}{C_{5}^{2} C_{6}^{\lceil r\rceil+1} \eta} \mathbf{P}^{0}\left(\tau_{B(0,1)}>1\right) \int_{B(0, r)} f(z) d z\right) \nu(x), \quad|x|>r+1 .
$$

(2) Let (A1.1) hold and suppose $\inf _{|y| \leq(r \vee 2)+1} f(y)>0$. Consider the following disjoint cases:

(i) (A1.2) holds and (A1.3) does not hold.

(ii) (A1.2) does not hold (and hence (A1.3) fails to hold).

Then in either of cases (i) and (ii) we have lim $\sup _{|x| \rightarrow \infty} \frac{f(x)}{\nu(x)}=\infty$.

Proof. First note that by $(X, \eta)$-harmonicity of $f$ in $\bar{B}(0, r)^{c}$ we have $f(x)=\mathbf{E}^{x}\left[e^{-\eta \tau_{B(x, 1)}} f\left(X_{\tau_{B(x, 1)}}\right)\right]$, for every $|x|>r+1$. Thus by the Ikeda-Watanabe formula (3.2), for every $|x|>r+1$

$$
\begin{aligned}
f(x) & \geq \int_{0}^{\infty} e^{-\eta t} \mathbf{P}^{x}\left(\tau_{B(x, 1)}>t\right) d t \inf _{y \in B(x, 1)} \int_{B(0, r)} f(z) \nu(z-y) d z \\
& \geq \frac{1}{C_{5}}\left(\int_{0}^{1} e^{-\eta t} d t \mathbf{P}^{0}\left(\tau_{B(0,1)}>1\right) \int_{B(0, r)} f(z) d z\right) g(|x|+1+r) \\
& \geq\left(\frac{1-e^{-\eta}}{C_{5}^{2} C_{6}^{\lceil r\rceil+1} \eta} \mathbf{P}^{0}\left(\tau_{B(0,1)}>1\right) \int_{B(0, r)} f(z) d z\right) \nu(x),
\end{aligned}
$$

which proves (1). We now show (2). When (A1.2) fails to hold (case (ii)), there exists a sequence $\left(r_{n}\right)_{n \in \mathbb{N}}$ such that $g\left(r_{n}-1\right) \geq n g\left(r_{n}\right)$. Notice that necessarily $r_{n} \rightarrow \infty$ as $n \rightarrow \infty$. Let $x_{n}=$ $\left(r_{n}, 0, \ldots, 0\right)$. With this, by following the argument as in (1) above, we get

$$
\begin{aligned}
f\left(x_{n}\right) & \geq \int_{0}^{\infty} e^{-\eta t} \int_{B\left(x_{n}, 1\right)} e^{-\eta t} p_{B\left(x_{n}, 1\right)}\left(t, x_{n}, z\right) d t \int_{B(0,(r \vee 2)+1)} f(y) \nu(z-y) d y d z \\
& \geq \frac{1}{C_{5}}\left(\int_{0}^{1} e^{-\eta t} d t \mathbf{P}^{0}\left(\tau_{B(0,1)}>1\right) \int_{B\left(\left(5 x_{n} / 2 r_{n}\right), 1 / 2\right)} f(y) d y\right) g\left(r_{n}-1\right) \\
& \geq n\left(\frac{1-e^{-\eta}}{C_{5} \eta} \mathbf{P}^{0}\left(\tau_{B(0,1)}>1\right) \inf _{|y| \leq(r \vee 2)+1} f(y)|B(0,1 / 2)|\right) g\left(r_{n}\right),
\end{aligned}
$$

for sufficiently large $n$. From this we easily see that $\lim _{n \rightarrow \infty} f\left(x_{n}\right) / \nu\left(x_{n}\right)=\infty$.

Consider now the case (i) and suppose that (A1.2) holds while (A1.3) does not. If (A1.3) breaks down, then there exists a divergent sequence $\left(s_{n}\right)_{n \in \mathbb{N}}$ such that for sufficiently large $n$

$$
\int_{\substack{\left|y-x_{n}\right|>1 \\|y|>1}} g\left(\left|x_{n}-y\right|\right) g(|y|) d y \geq n g\left(\left|x_{n}\right|\right), \quad n \in \mathbf{N}, \quad \text { where } \quad x_{n}=\left(s_{n}, 0, \ldots, 0\right) .
$$


By the facts $g(|y|) \leq g(1)<\infty,|y| \geq 1$, and $\inf _{|y| \leq r+1} f(y)>0$, there exists $c=c(r)$ such that $g(|y|) \leq c f(y)$, for $1 \leq|y| \leq r+1$. This implies jointly with (1) that

$$
f(y) \geq c_{1} g(|y|), \quad|y| \geq 1, \quad \text { for some } c_{1}>0 .
$$

Proceeding now in the same way as in (1), by making use of (3.3) and (A1.2) we get

$$
\begin{aligned}
f\left(x_{n}\right) & \geq \frac{c_{1}}{C_{5}} \int_{0}^{\infty} \int_{B\left(x_{n}, 1 / 2\right)} e^{-\eta t} p_{B\left(x_{n}, 1 / 2\right)}\left(t, x_{n}, z\right) d t \int_{\substack{\left|y-x_{n}\right|>1 / 2 \\
|y|>1}} g(|z-y|) g(|y|) d y d z \\
& \geq \frac{c_{2}}{C_{5}} \int_{0}^{1} e^{-\eta t} d t \mathbf{P}^{0}\left(\tau_{B(0,1 / 2)}>1\right) \int_{\substack{\left|y-x_{n}\right|>1 \\
|y|>1}} g\left(\left|x_{n}-y\right|\right) g(|y|) d y d z \\
& \geq n\left(\frac{c_{2}}{C_{5}} \int_{0}^{1} e^{-\eta t} d t \mathbf{P}^{0}\left(\tau_{B(0,1 / 2)}>1\right)\right) g\left(\left|x_{n}\right|\right),
\end{aligned}
$$

for sufficiently large $n$. Similarly as above, this completes the proof in the case (i).

\subsection{Uniform estimate for suprema of functions harmonic in balls}

In this subsection we derive a uniform upper bound for functions that are regular $(X, \eta)$-harmonic in large balls, which will be a basic technical tool in what follows. It can be obtained as an adaptation of the strong estimates of [10] to our framework.

For $s_{1} \geq 1$ and $s_{2} \geq 2 s_{1}$ define

$$
h_{1}\left(X, s_{1}, s_{2}\right)=K_{2}^{X}\left(s_{1}, s_{2}, \infty\right)\left[C_{3}\left(X, \frac{s_{1}}{16}\right)\left(C_{13}\left(X, s_{1}\right)\left|B\left(0, s_{1}\right)\right|+\mathbf{E}^{0}\left[\tau_{B\left(0,2 s_{1}\right)}\right)\right)+1\right]
$$

and

$$
h_{2}\left(X, s_{1}\right)=C_{3}\left(X, \frac{s_{1}}{16}\right)\left[C_{3}\left(X, s_{1}\right) C_{13}\left(X, s_{1}\right)+\mathbf{E}^{0}\left[\tau_{B\left(0,2 s_{1}\right)}\right] \sup _{|y| \geq \frac{s_{1}}{4}} \nu(y)\right]+\sup _{|y| \geq \frac{s_{1}}{16}} \nu(y),
$$

where

$$
C_{13}\left(X, s_{1}\right):=K_{3}^{X}\left(s_{1}\right)+\frac{\mathbf{E}^{0}\left[\tau_{B\left(0,2 s_{1}\right)}\right]}{\left|B\left(0, \frac{s_{1}}{4}\right)\right|}\left(K_{2}^{X}\left(\frac{s_{1}}{4}, \frac{s_{1}}{2}, s_{1}\right)\right)^{2} .
$$

It is of key importance that neither $h_{1}$ nor $h_{2}$ depends on $x, \eta$ and the function $f$. With the above notation we have the following.

Lemma 3.1. Let $\left(X_{t}\right)_{t \geq 0}$ be a Lévy process with Lévy-Khintchin exponent $\psi$ given by (2.1)-(2.2) such that Assumptions (A1.1)-(A1.2) and (A3) hold, and let $s_{1} \geq 1$ and $s_{2} \geq 2 s_{1}$. Then for every $\eta>0$ and every non-negative function $f$ on $\mathbf{R}^{d}$ which is regular $(X, \eta)$-harmonic in a ball $B\left(x, s_{1}\right)$, $x \in \mathbf{R}^{d}$, we have

$$
f(y) \leq \frac{1}{\eta}\left(h_{1}\left(X, s_{1}, s_{2}\right) \int_{|z-x|>s_{2}} f(z) \nu(z-x) d z+h_{2}\left(X, s_{1}\right) \int_{\frac{s_{1}}{8}<|x-z| \leq s_{2}} f(z) d z\right), \quad|y-x|<\frac{s_{1}}{32} .
$$

Proof. The claimed bound is a version of the upper estimate in (3.3) of [10, Lem. 3.2], but the constant in that bound is not suitable for our purposes here and (3.4) does not follow from the statement in the cited paper. However, the required form of constants can be obtained from the original statement with some extra work, and in this proof we keep to the notation of [10] for the reader's convenience. 
Note that by conditions (2.2), (A1.1)-(A1.2) and (A3) above all of the Assumptions A, B, C, D in [10] hold. Let $s_{1} \geq 1$ and $s_{2} \geq 2 s_{1}$. First we show that a version of the estimate [10, (3.7)] holds with $R=s_{1}, q=s_{1} / 2, p=s_{1} / 4$ and $r=s_{1} / 8$, i.e.,

$$
f(y) \leq \int_{|z-x| \geq \frac{s_{1}}{2}} f(z) \pi_{s_{1}, s_{2}}(z-x) d z, \quad|y-x|<\frac{s_{1}}{8}
$$

where

$\pi_{s_{1}, s_{2}}(z-x)=\left\{\begin{array}{lr}C_{3}\left(X, s_{1}\right) C_{13}\left(X, s_{1}\right)+\mathbf{E}^{0}\left[\tau_{B\left(0,2 s_{1}\right)}\right] \sup _{|y| \geq \frac{s_{1}}{4}} \nu(y) \quad \text { for } \quad \frac{s_{1}}{2} \leq|z-x| \leq s_{2}, \\ K_{2}^{X}\left(s_{1}, s_{2}, \infty\right)\left(C_{13}\left(X, s_{1}\right)\left|B\left(0, s_{1}\right)\right|+\mathbf{E}^{0}\left[\tau_{B\left(0,2 s_{1}\right)}\right]\right) \nu(z-x) & \text { for }|z-x|>s_{2} .\end{array}\right.$

This can be seen by following through the argument in [10, Th. 3.4]. First observe that the constant $c_{(2.7)}(x, p, q)$ can be "localized" in space, i.e., its value actually depends on the position $z$ appearing in $\nu(z-x)$ and $\nu(z-y)$ when $|x-y|<p=s_{1} / 4$. Indeed, we have

$$
c_{(2.7)}(x, p, q)=\left\{\begin{array}{lll}
K_{2}^{X}\left(s_{1} / 4, s_{1} / 2, s_{1}\right) & \text { for } \quad \frac{s_{1}}{2} \leq|z-x| \leq s_{1}, \\
K_{2}^{X}\left(s_{1} / 4, s_{1}, s_{2}\right) & \text { for } \quad s_{1}<|z-x| \leq s_{2}, \\
K_{2}^{X}\left(s_{1} / 4, s_{2}, \infty\right) & \text { for } \quad|z-x|>s_{2} .
\end{array}\right.
$$

Moreover, recall that $K_{2}^{X}\left(v, s_{2}, \infty\right)$ is non-decreasing in $v \in\left(0, s_{2}\right)$ (we emphasize that in our space-homogeneous case none of the constants depends on $x$ ). Also, we see that in our setting $c_{(2.9)}(x, R) \leq \mathbf{E}^{0}\left[\tau_{B\left(0,2 s_{1}\right)}\right]$ and $c_{(2.10)}(x, r, p, R) \leq K_{3}^{X}\left(s_{1}\right)$. By this and (3.6) we can directly check that in $\left[10\right.$, Lem. 4.5] we have $c_{(4.17)}(x, r, p, q, R) \leq C_{13}\left(X, s_{1}\right)$, i.e., the constant $c_{(2.7)}(x, p, q)$ appearing in the estimates is equal to $K_{2}^{X}\left(s_{1} / 4, s_{1} / 2, s_{1}\right)$. With this, we can now verify that the statement of [10, Lem. 4.9] stays valid with the kernel $\tilde{\pi}_{\psi}(z)$ replaced by

$\tilde{\pi}_{s_{1}, s_{2}}(z-x)=\left\{\begin{array}{lll}C_{13}\left(X, s_{1}\right) \delta+\mathbf{E}^{0}\left[\tau_{B\left(0,2 s_{1}\right)}\right] \sup _{|w| \geq \frac{s_{1}}{4}} \nu(w) & \text { for } & \frac{s_{1}}{2} \leq|z-x| \leq s_{2}, \\ K_{2}^{X}\left(s_{1}, s_{2}, \infty\right)\left(C_{13}\left(X, s_{1}\right)\left|B\left(0, s_{1}\right)\right|+\mathbf{E}^{0}\left[\tau_{B\left(0,2 s_{1}\right)}\right]\right) \nu(z-x) & \text { for }|z-x|>s_{2},\end{array}\right.$

in $[10,(4.21)]$. To do that, recall the specific choices $R=s_{1}, q=s_{1} / 2, p=s_{1} / 4, r=s_{1} / 8$, and observe that $[10,(4.22)]$ holds with the constant $C_{13}\left(X, s_{1}\right) \delta$. We can now continue similarly as in the second part of the proof of [10, Lem.4.9]. For $|w-x| \leq s_{1} / 8$ and $z \in V^{c}$ such that $s_{1} / 2 \leq|z-x| \leq s_{2}$ we have

$$
\int_{V \cap B\left(x, s_{1} / 4\right)^{c}} G_{\psi}(w, y) \nu(y-z) d y=\int_{V \cap B\left(x, s_{1} / 4\right)^{c}} G_{\psi}(y, w) \nu(y-z) d y \leq C_{13}\left(X, s_{1}\right) \delta
$$

and

$$
\int_{B\left(x, s_{1} / 4\right)} G_{\psi}(w, y) \nu(y-z) d y \leq \sup _{|v| \geq \frac{s_{1}}{4}} \nu(v) \mathbf{E}^{w}\left[\tau_{B\left(x, s_{1}\right)}\right] \leq \sup _{|v| \geq \frac{s_{1}}{4}} \nu(v) \mathbf{E}^{0}\left[\tau_{B\left(0,2 s_{1}\right)}\right] .
$$

(Since we use here the original notation, $V$ now means the set defined in [10, (4.2)], and $G_{\psi}(w, y)$ is the potential kernel as in [10, p.492].) Moreover, for $|z-x|>s_{2}$, in which case necessarily $z \in V^{c}$, we get

$$
\begin{aligned}
\int_{V \cap B\left(x, s_{1} / 4\right)^{c}} G_{\psi}(w, y) \nu(y-z) d y & \leq C_{13}\left(X, s_{1}\right) \nu\left(B\left(x, s_{1}\right)-z\right) \\
& \leq C_{13}\left(X, s_{1}\right) K_{2}^{X}\left(s_{1}, s_{2}, \infty\right)\left|B\left(0, s_{1}\right)\right| \nu(x-z)
\end{aligned}
$$


and

$$
\begin{aligned}
\int_{B\left(x, s_{1} / 4\right)} G_{\psi}(w, y) \nu(y, z) d y & \leq K_{2}^{X}\left(s_{1}, s_{2}, \infty\right) \mathbf{E}^{w}\left[\tau_{B\left(x, s_{1}\right)}\right] \nu(x-z) \\
& \leq K_{2}^{X}\left(s_{1}, s_{2}, \infty\right) \mathbf{E}^{0}\left[\tau_{B\left(0,2 s_{1}\right)}\right] \nu(x-z) .
\end{aligned}
$$

Combining these estimates, we conclude similarly as in [10, Lem. 4.9] that the claimed bound holds with the kernel $\tilde{\pi}_{\psi}(z)$ replaced by $\tilde{\pi}_{s_{1}, s_{2}}(z-x)$. Since $\left.\varrho=\varrho(\bar{B}(x, q), B(x, R))\right) \leq C_{3}\left(X, s_{1}\right)$, the proof of (3.5) can be completed in the same way as in [10, Th. 3.4].

To complete the proof of the lemma, we follow the argument leading from (a) to (b) in the proof of $[10$, Lem. 3.2]. By regular $(X, \eta)$-harmonicity of $f$ we get

$$
\begin{aligned}
& f(y)=\mathbf{E}^{y}\left[e^{-\eta \tau_{B}\left(x, \frac{s_{1}}{16}\right)} f\left(X_{\left.\tau_{B\left(x, \frac{s_{1}}{16}\right.}\right)}\right) ; X_{\tau_{B\left(x, \frac{s_{1}}{16}\right)}} \in B\left(x, \frac{s_{1}}{8}\right)\right] \\
& +\mathbf{E}^{y}\left[e^{-\eta \tau_{B}\left(x, \frac{s_{1}}{16}\right)} f\left(X_{\tau_{B\left(x, \frac{s_{1}}{16}\right)}}\right) ; X_{\tau_{B\left(x, \frac{s_{1}}{16}\right)}} \in B\left(x, \frac{s_{1}}{8}\right)^{c}\right]=\mathrm{I}+\mathrm{II},
\end{aligned}
$$

whenever $|y-x|<s_{1} / 32$. By the Ikeda-Watanabe formula (3.2) we furthermore have

$$
\begin{aligned}
& \mathrm{II} \leq \mathbf{E}^{y}\left[\int_{0}^{\tau B\left(x, \frac{s_{1}}{16}\right)} e^{-\eta t} d t\right]\left(\sup _{|w|>\frac{s_{1}}{16}} \nu(w) \int_{\frac{s_{1}}{8} \leq|z-x| \leq s_{2}} f(z) d z\right. \\
& \left.+K_{2}^{X}\left(\frac{s_{1}}{16}, s_{2}, \infty\right) \int_{|z-x|>s_{2}} f(z) \nu(z-x) d z\right) \\
& \leq \frac{1}{\eta}\left(\sup _{|w|>\frac{s_{1}}{16}} \nu(w) \int_{\frac{s_{1}}{8} \leq|z-x| \leq s_{2}} f(z) d z+K_{2}^{X}\left(s_{1}, s_{2}, \infty\right) \int_{|z-x|>s_{2}} f(z) \nu(z-x) d z\right) .
\end{aligned}
$$

Similarly, by the version of [10, Lem. 3.1], see also [10, Ex. 5.9], for the subprocess of $\left(X_{t}\right)_{t \geq 0}$ corresponding to the multiplicative functional $M_{t}=e^{-\eta t}$ and (3.5), we obtain

$$
\begin{aligned}
\mathrm{I} & \leq \mathbf{E}^{y}\left[e^{\left.-\eta \tau(x), \frac{s_{1}}{16}\right)} ; X_{\tau_{B}\left(x, \frac{s_{1}}{16}\right)} \in B\left(x, \frac{s_{1}}{8}\right)\right] \sup _{|y-x|<\frac{s_{1}}{8}} f(y) \\
& \leq \frac{C_{3}\left(X, \frac{s_{1}}{16}\right)}{\eta} \int_{|z-x|>\frac{s_{1}}{2}} f(z) \pi_{s_{1}, s_{2}}(z-x) d z .
\end{aligned}
$$

By putting together the estimates of I and II above (3.4) follows.

Remark 3.1. The estimate (3.4) is somewhat laborious (with no apparent ways to simplify the argument), however, it has the correct structure required by the applications of Lemma 3.1 in the next two subsections. In Lemma 3.2 following below, (3.4) will be iterated infinitely many times resulting in a self-improving estimate which leads to an upper bound of harmonic functions controlled by $\nu$ alone. To realise this for arbitrarily small $\eta>0$, we need to ensure that both prefactors $h_{1}, h_{2}$ in (3.4) are small enough. This requires to work with a sufficiently large domain of harmonicity (large $s_{1}>0$ ) and introduce the additional control parameters $r_{2} \gg r_{1}$ and $r_{3}>r_{2}$. Another significant difference between (3.4) and the original bound in [10] is that our function $K_{2}^{X}$ appearing in the expression of $C_{13}$ depends only on the form of $\nu(y)$ for $s_{1} / 4 \leq|y| \leq s_{1}+s_{1} / 4$ but not for $|y| \geq r_{2}$. The use of this construction will get fully transparent in the proof of our main technical result in Theorem 3.3, where we deal with Lévy measures fast decaying at infinity. 


\subsection{Upper bound for functions harmonic at infinity}

The next lemma is the first key technical result of this paper and it will be fundamental for our investigations below. Recall that the functions $K_{1}^{X}, K_{2}^{X}$ are defined in (2.12)-(2.13).

Lemma 3.2. Let $\eta>0$ and $\left(X_{t}\right)_{t \geq 0}$ be a Lévy process with Lévy-Khintchin exponent $\psi$ given by (2.1)-(2.2) such that Assumptions (A1)-(A3) hold. Moreover, suppose that there exist $r_{1} \geq 1$, $r_{2} \geq 2 r_{1}$ and $r_{3}>r_{2}$ such that

$$
2 C_{5}^{4} h_{1}\left(X, r_{1}, r_{2}\right) K_{1}^{X}\left(r_{2}\right)+h_{2}\left(X, r_{1}\right)\left|B\left(0, r_{2}\right)\right| K_{2}^{X}\left(r_{2}, r_{3}, \infty\right)<\eta .
$$

Then for every bounded function $f \geq 0$ which is $(X, \eta)$-harmonic in $\bar{B}(0, r)^{c}$ for some $r>0$, we have

$$
f(x) \leq C_{14}\|f\|_{\infty} \nu(x), \quad|x| \geq R+1,
$$

with $R=R(X, \eta):=\left(r+r_{1}\right) \vee r_{3}$ and

$$
C_{14}=C_{14}(X, \eta):=\frac{C_{5}^{2} C_{6}^{\lceil R\rceil}\left(1+C_{8}\right)\left(h_{1}\left(X, r_{1}, r_{2}\right)+h_{2}\left(X, r_{1}\right) \frac{1}{\inf _{|y| \leq r_{2}} \nu(y)}\right)|B(0, R)|}{\eta-h_{1}\left(X, r_{1}, r_{2}\right) K_{1}^{X}\left(r_{2}\right)-h_{2}\left(X, r_{1}\right)\left|B\left(0, r_{2}\right)\right| K_{2}^{X}\left(r_{2}, r_{3}, \infty\right)} .
$$

Proof. Fix $\eta, r>0, r_{1}>1, r_{2} \geq 2 r_{1}$ and $r_{3}>r_{2}$ as in the statement. Denote $R:=\left(r+r_{1}\right) \vee r_{3}$. By $(X, \eta)$-harmonicity of the function $f$ in $\bar{B}(0, r)^{c}$ we have that $f(y)=\mathbf{E}^{y}\left[e^{-\eta \tau_{B\left(x, r_{1}\right)}} f\left(X_{\tau_{B\left(x, r_{1}\right)}}\right]\right.$, $y \in B\left(x, r_{1}\right)$, whenever $|x|>R$. Thus by Lemma 3.1 and (2.11), for every $|x| \geq R+1$ we obtain

$$
\begin{aligned}
f(x) \leq & \frac{1}{\eta}\left(h_{1}\left(X, r_{1}, r_{2}\right) \int_{|z-x|>r_{2}} f(z) \nu(z-x) d z+h_{2}\left(X, r_{1}\right) \int_{\frac{r_{1}}{8}<|x-z| \leq r_{2}} f(z) d z\right) \\
= & \frac{1}{\eta}\left(h_{1}\left(X, r_{1}, r_{2}\right) \int_{\substack{|z-x|>r_{2} \\
|z| \leq R+1}} f(z) \nu(z-x) d z+h_{2}\left(X, r_{1}\right) \int_{\frac{r_{1}}{8}<|x-z| \leq r_{2}} f(z) d z\right) \\
& +\frac{1}{|z| \leq R+1}\left(h_{1}\left(X, r_{1}, r_{2}\right) \int_{\substack{|z-x|>r_{2} \\
|z|>R+1}} f(z) \nu(z-x) d z+h_{2}\left(X, r_{1}\right) \int_{\frac{r_{1}}{8}<|x-z| \leq r_{2}} f(z) d z\right) \\
\leq & \frac{1+C_{8}}{\eta}\left(h_{1}\left(X, r_{1}, r_{2}\right)+\frac{h_{2}\left(X, r_{1}\right)}{\inf |y| \leq r_{2} \nu(y)}\right)\|f\|_{\infty} \int_{|z| \leq R} \nu(x-z) d z \\
& +\frac{1}{\eta}\left(h_{1}\left(X, r_{1}, r_{2}\right) \int_{\substack{|z-x|>r_{2} \\
|z|>R+1}} f(z) \nu(z-x) d z+h_{2}\left(X, r_{1}\right) \int_{\frac{r_{1}<|x-z| \leq r_{2}}{8}|z|>R+1} f(z) d z\right) .
\end{aligned}
$$

Furthermore, by (A1.1)-(A1.2),

$$
f(x) \leq c\|f\|_{\infty} \nu(x)+\frac{1}{\eta}\left(h_{1}\left(X, r_{1}, r_{2}\right) \int_{\substack{|z-x|>r_{2} \\|z|>R+1}} f(z) \nu(z-x) d z+h_{2}\left(X, r_{1}\right) \int_{\frac{r_{1}}{8}<|x-z| \leq r_{2}} f(z) d z\right),
$$

where

$$
c:=\frac{C_{5}^{2} C_{6}^{\lceil R\rceil}\left(1+C_{8}\right)}{\eta}\left(h_{1}\left(X, r_{1}, r_{2}\right)+h_{2}\left(X, r_{1}\right) \frac{1}{\inf _{|y| \leq r_{2}} \nu(y)}\right)|B(0, R)| .
$$

This gives

$$
f(x) \leq\|f\|_{\infty}\left(c \nu(x)+c_{1}\right), \quad|x| \geq R+1,
$$

with

$$
c_{1}=\frac{1}{\eta}\left[h_{1}\left(X, r_{1}, r_{2}\right) \nu\left(B\left(0, r_{2}\right)^{c}\right)+h_{2}\left(X, r_{1}\right)\left|B\left(0, r_{2}\right)\right|\right]
$$


Also, write

$$
c_{2}=\frac{1}{\eta}\left(h_{1}\left(X, r_{1}, r_{2}\right) K_{1}^{X}\left(r_{2}\right)+h_{2}\left(X, r_{1}\right)\left|B\left(0, r_{2}\right)\right| K_{2}^{X}\left(r_{2}, r_{3}, \infty\right)\right) .
$$

Assumption (3.7) and Lemma 2.1 imply that $c_{1} \vee c_{2}<1$, which will be essential in what follows.

We now show that for every $p \in \mathbf{N}$

$$
f(x) \leq c\|f\|_{\infty} \sum_{i=1}^{p} c_{2}^{i-1} \nu(x)+\|f\|_{\infty} c_{1}^{p}, \quad|x| \geq R+1 .
$$

Notice that if this holds, then by taking the limit $p \rightarrow \infty$ it follows that

$$
f(x) \leq \frac{c}{1-c_{2}}\|f\|_{\infty} \nu(x), \quad|x| \geq R+1,
$$

which is the bound stated in the lemma.

To prove (3.10) we make induction on $p \in \mathbf{N}$. First observe that (3.9) is just (3.10) for $p=1$. Suppose now that (3.10) is true for $p-1 \in \mathbf{N}$. By (3.8) and the induction hypothesis we see for all $|x| \geq R+1$ that

$$
\begin{aligned}
f(x) \leq & c\|f\|_{\infty} \nu(x)+\frac{c\|f\|_{\infty}}{\eta} \sum_{i=1}^{p-1} c_{2}^{i-1}\left(h_{1}\left(X, r_{1}, r_{2}\right)\|f\|_{\infty} \int_{\substack{|z-x|>r_{2} \\
|z|>R+1}} \nu(z) \nu(z-x) d z\right. \\
& \left.+h_{2}\left(X, r_{1}\right)\left|B\left(0, r_{2}\right)\right| \sup _{|z-x| \leq r_{2}} \nu(z)\right) \\
+ & \frac{\|f\|_{\infty} c_{1}^{p-1}}{\eta}\left(h_{1}\left(X, r_{1}, r_{2}\right) \nu\left(B\left(0, r_{2}\right)^{c}\right)+h_{2}\left(X, r_{1}\right)\left|B\left(0, r_{2}\right)\right|\right) .
\end{aligned}
$$

Applying (2.12) to the integral in the second summand and using the definition of the constants $c_{1}, c_{2}$ gives

$$
\begin{aligned}
f(x) \leq & c\|f\|_{\infty} \nu(x) \\
& +c\|f\|_{\infty} \sum_{i=1}^{p-1} \frac{c_{2}^{i-1}}{\eta}\left[h_{1}\left(X, r_{1}, r_{2}\right) K_{1}^{X}\left(r_{2}\right)+h_{2}\left(X, r_{1}\right)\left|B\left(0, r_{2}\right)\right| K_{2}^{X}\left(r_{2}, r_{3}, \infty\right)\right] \nu(x) \\
& +\|f\|_{\infty} c_{1}^{p-1} \frac{h_{1}\left(X, r_{1}, r_{2}\right) \nu\left(B\left(0, r_{2}\right)^{c}\right)+h_{2}\left(X, r_{1}\right)\left|B\left(0, r_{2}\right)\right|}{\eta} \\
\leq & c\|f\|_{\infty} \sum_{i=1}^{p} c_{2}^{i-1} \nu(x)+\|f\|_{\infty} c_{1}^{p},
\end{aligned}
$$

which completes the proof.

The power of Lemma 3.2 depends on the verifiability of condition (3.7). As it will turn out, whether the Lévy intensity has a slow or quick decay will make a difference, and the latter case is more difficult.

First we apply Lemma 3.2 to intensities which are slowly decaying in the sense of the following assumption:

$$
\begin{aligned}
& \text { there exists } C_{15}>0 \text { such that for all } r \geq 1 \text { we have } \\
& \nu(x-y) \leq C_{15} \nu(x) \text { whenever }|y| \leq r \text { and }|x| \geq 2 r
\end{aligned}
$$

This property typically holds for functions polynomially decaying at infinity. It is straightforward to check that under (3.11) we have

$$
K_{1}^{X}(s) \leq C_{5}^{2} C_{15} \nu\left(B(0, s)^{c}\right), \quad \text { for any } s \geq 1,
$$


and

$$
K_{2}^{X}\left(s_{1}, s_{2}, s_{3}\right) \leq C_{15}, \quad \text { for every } s_{1} \geq 1, s_{2} \geq 2 s_{1} \text { and } s_{2}<s_{3} \leq \infty .
$$

This immediately gives that under assumption (3.11) conditions (2.15)-(2.16) hold automatically with $\kappa_{1}=2$ and $\kappa_{2}=C_{15}$. By this fact, in the following theorem our standard set of assumptions (2.15), (2.16), (2.19) simplifies to (3.11) and (2.19).

Theorem 3.2. Let $\left(X_{t}\right)_{t \geq 0}$ be a Lévy process with Lévy-Khintchin exponent $\psi$ given by (2.1)-(2.2) such that Assumptions (A1)-(A3) hold. Moreover, suppose that conditions (3.11) and (2.19) hold. Then for every $\eta>0$ and every bounded non-negative function $f$ which is $(X, \eta)$-harmonic in $\bar{B}(0, r)^{c}$ for some $r>0$, there exist $C_{16}=C_{16}(X, \eta)$ and $R=R(X, \eta)$ such that

$$
f(x) \leq C_{16}\|f\|_{\infty} \nu(x), \quad|x| \geq R .
$$

Proof. Notice that whenever $s \geq 4$, under (3.11) we have $K_{2}^{X}(s / 4, s / 2, s) \leq C_{15}, K_{2}^{X}(s, 2 s, \infty) \leq$ $C_{15}$, and $K_{2}^{X}(2 s, 4 s, \infty) \leq C_{15}$. Thus by (2.8), (2.10) and (2.19) we also have $h_{1}(X, s, 2 s) \leq c_{1}$ for the same $s$. This and (3.12) jointly imply that

$$
h_{1}(X, s, 2 s) K_{1}^{X}(2 s) \leq c_{1} K_{1}^{X}(2 s) \rightarrow 0 \quad \text { as } \quad s \rightarrow \infty .
$$

Similarly, by (2.8), (2.10), (2.19) and the fact that $g(s) s^{d} \rightarrow 0$ as $s \rightarrow \infty$, we get

$$
s^{d} h_{2}(X, s) K_{2}^{X}(2 s, 4 s, \infty) \rightarrow 0 \quad \text { as } \quad s \rightarrow \infty .
$$

To complete the proof, observe that for every $\eta>0$ there exists $s \geq 4$ such that condition (3.7) holds with $r_{1}=s, r_{2}=2 r_{1}=2 s$ and $r_{3}=4 s>r_{2}$. The claimed bound follows from Lemma 3.2 .

\subsection{Upper bound for the Laplace transform of the first hitting time of a ball}

A specific harmonic function to which we need to apply the results of the previous subsection in order to study the decay of eigenfunctions is of the type

$$
f(x)=\left\{\begin{array}{lll}
\mathbf{E}^{x}\left[e^{-\eta \tau_{\bar{B}(0, r)^{c}}}\right] & \text { for } & |x|>r, \\
\mathbf{1}_{\{|x| \leq r\}}(x) & \text { for } & |x| \leq r .
\end{array}\right.
$$

Here we use the standard convention that $1 / \infty=0$.

Condition (3.11) fails to hold for Lévy measures which are lighter than polynomial at infinity. Although Theorem 3.2 cannot be directly extended to this class of processes, the structure involving the functions $h_{1}, h_{2}$ in (3.4) allows to use Lemma 3.2 also in this case. However, for such Lévy measures the function $K_{2}^{X}\left(s_{1} / 4, s_{1} / 2, s_{1}\right)$ appearing in $C_{13}\left(X, s_{1}\right)$ typically diverges faster than polynomially for large $s$, and $s_{1}^{d} h_{2}\left(X, s_{1}\right)$ does not vanish as $s_{1} \rightarrow \infty$. This problem occurs when, for instance, $\nu(x)$ decays like $e^{-|x|^{\beta}}$, for some $\beta \in(0,1)$. This difficulty persists even when taking a fixed positive number $s_{0}$ instead of proportionally increasing the radius $s_{1} / 4$ to replace $K_{2}^{X}\left(s_{1} / 4, s_{1} / 2, s_{1}\right)$ by $K_{2}^{X}\left(s_{0}, s_{1} / 2, s_{1}\right)$. (Indeed, for Lévy densities satisfying (A1) it is always true that we can choose a $C=C\left(s_{0}\right)$ such that $K_{2}^{X}\left(s_{0}, s_{1} / 2, s_{1}\right) \leq C$ for large $s_{1}>0$, however, we see that in this case $C_{3}\left(X, s_{1} / 16\right)$ in $h_{2}\left(X, s_{1}\right)$ must be replaced by a strictly positive constant independent of $s_{1}$ and again the expression $s_{1}^{d} h_{2}\left(X, s_{1}\right)$ cannot vanish as $s_{1} \rightarrow \infty$.) Therefore, in Theorem 3.3 below giving the upper bound of (3.13) for Lévy densities which are light at infinity we choose the strategy to keep all the radii in (3.4) proportional to $s_{1}$. To get this result, we will use an argument based on the domination of $\nu$ by a carefully constructed family of jump intensities. Specifically, for any small $\eta>0$ we will choose a sufficiently large radius of harmonicity $s_{1}$ and construct a Lévy process 
with jump intensity $\nu^{s_{1}}$ to which we can effectively apply Lemma 3.2 and which has the following properties:

(1) $\nu^{s_{1}}$ dominates $\nu$ inside $B\left(0, s_{1}\right)$ and agrees with $\nu$ outside $B\left(0, s_{2}\right)$ for sufficiently large $s_{2} \gg s_{1}$ proportional to $s_{1}$, but the difference $\nu^{s_{1}}-\nu$ is relatively small with respect to $\eta$.

(2) the function (3.13) corresponding to $\nu$ is dominated by that of $\nu^{s_{1}}$ with $\eta / 2$ at infinity.

To achieve this, we make use of the following comparison scheme.

Proposition 3.1. Let $\left(X_{t}^{(1)}\right)_{t \geq 0}$ and $\left(X_{t}^{(2)}\right)_{t \geq 0}$ be two Lévy processes with characteristic exponents $\psi^{(1)}, \psi^{(2)}$ as in (2.1), the same diffusion coefficient $A$, and Lévy measures $\nu^{(1)}, \nu^{(2)}$ satisfying (2.2) and such that $\sigma:=\nu^{(2)}-\nu^{(1)}$ is a non-negative finite measure. Denote $|\sigma|:=\sigma\left(\mathbf{R}^{d} \backslash\{0\}\right)$. Then the following hold.

(1) For every $t>0$ and almost every $x \in \mathbf{R}^{d}$, we have

$$
e^{-|\sigma| t} p^{(1)}(t, x) \leq p^{(2)}(t, x) \leq e^{-|\sigma| t} p^{(1)}(t, x)+t \sup _{z \in \mathbf{R}^{d}} \sigma(z)
$$

where $p^{(1)}(t, x), p^{(2)}(t, x)$ are the transition densities of the processes $\left(X_{t}^{(1)}\right)_{t \geq 0},\left(X_{t}^{(2)}\right)_{t \geq 0}$, respectively, and $\sigma(x):=\nu^{(2)}(x)-\nu^{(1)}(x)$ for $x \neq 0, \sigma(0):=0$.

(2) For every $\eta>|\sigma|$ and almost every $x \in \mathbf{R}^{d}$, we have $G_{1}^{\eta}(x) \leq G_{2}^{\eta-|\sigma|}(x)$, where $G_{i}^{\eta}(x)=$ $\int_{0}^{\infty} e^{-\eta t} p^{(i)}(t, x) d t, i=1,2$, are the $\eta$-potential kernels of the two processes, respectively.

(3) If there exist $C_{17} \geq 1$ and $R>0$ such that $p^{(1)}(t, x) \leq C_{17} p^{(1)}(t, y)$ for every $|x| \geq|y| \geq R$, $|x-y| \leq 1$, and $t>0$, then for every $\eta>|\sigma|$ and $r>0$ there exists a constant $C_{18}=$ $C_{18}\left(X^{(1)}, X^{(2)}, \eta, r\right)$ such that for every $|x|>3 r+R$ we have

$$
\mathbf{E}^{x}\left[e^{-\eta \tau_{r}^{(1)}}\right] \leq C_{17}^{2\lceil 2 r\rceil} C_{18} \mathbf{E}^{x-x_{2 r}}\left[e^{-(\eta-|\sigma|) \tau_{r}^{(2)}}\right]
$$

where $x_{r}:=(r /|x|) x$ and $\tau_{r}^{i}:=\inf \left\{t>0: X_{t}^{(i)} \in \bar{B}(0, r)\right\}, i=1,2$.

Proof. First consider (1). Recall that the compound Poisson measure corresponding to $\sigma$ is given by $e^{t \sigma}=e^{-t|\sigma|} \sum_{n=0}^{\infty} \frac{t^{n} \sigma^{n *}}{n !}, t>0$. Since $\nu^{(2)}=\nu^{(1)}+\sigma$, we can write

$p^{(2)}(t, x)=p^{(1)}(t, \cdot) * \exp (t \sigma)(x)=p^{(1)}(t, x) e^{-t|\sigma|}+e^{-t|\sigma|} \sum_{n=1}^{\infty} \frac{t^{n} p^{(1)}(t, \cdot) * \sigma^{n *}(x)}{n !}, t>0$, a.e. $x \in \mathbf{R}^{d}$.

From this we see that the first inequality in (1) holds. To show the second, it suffices to estimate the sum at the right hand side. We have

$$
p^{(1)}(t, \cdot) * \sigma^{n *}(x)=\int_{\mathbf{R}^{d}} \sigma^{n *}(x-y) p(t, y) d y \leq \sup _{z \in \mathbf{R}^{d}} \sigma^{n *}(z) \leq|\sigma|^{n-1} \sup _{z \in \mathbf{R}^{d}} \sigma(z),
$$

for every $t>0, n \in \mathbf{N}$ and almost every $x \in \mathbf{R}^{d}$. Hence,

$$
p^{(2)}(t, x) \leq e^{-t|\sigma|} p^{(1)}(t, x)+t \sup _{z \in \mathbf{R}^{d}} \sigma(z) e^{-t|\sigma|} \sum_{n=1}^{\infty} \frac{t^{n-1}|\sigma|^{n-1}}{n !} \leq e^{-t|\sigma|} p^{(1)}(t, x)+t \sup _{z \in \mathbf{R}^{d}} \sigma(z),
$$

for all $t>0$ and almost every $x \in \mathbf{R}^{d}$, which proves the claim. Both inequalities in (1) directly extend to the case when $A$ is non-zero. Indeed, then for every fixed $t>0$ the resulting transition density is a convolution of the Gaussian measure and the kernel corresponding to the jump part of the process, for which the required bounds are proven above.

Assertion (2) is a direct consequence of the first inequality in (1), since for every $\eta>0$ and almost every $x \in \mathbf{R}^{d}$, we have $G_{i}^{\eta}(x)=\int_{0}^{\infty} e^{-\eta t} p^{(i)}(t, x) d t, i=1,2$. 
Now we show (3). Denote by $\mu_{B\left(x_{0}, r\right)}^{\eta,(i)}(d x)$ the $\eta$-capacitory measure of the ball $B\left(x_{0}, r\right), x_{0} \in \mathbf{R}^{d}$, for the process $\left(X_{t}^{(i)}\right)_{t \geq 0}$ (see e.g. [5, Sect. 2, Ch. II]). It is known to be a Radon measure with support contained in $\bar{B}\left(x_{0}, r\right)$. By [5, Th. 7 and pp.51-52], for every $\eta>0, x \in \mathbf{R}^{d}$ and $r>0$ we have

$$
\mathbf{E}^{x}\left[e^{-\eta \tau_{\bar{B}\left(x_{0}, r\right)^{c}}^{(i)}}\right]=\int_{\bar{B}\left(x_{0}, r\right)} G_{i}^{\eta}(x-y) \mu_{B\left(x_{0}, r\right)}^{\eta,(i)}(d y), \quad i=1,2 .
$$

When $x_{0}=0$, we write $\tau_{r}^{(i)}$ and $\mu_{r}^{\eta,(i)}(d x)$ for a shorthand. From here, for every $\eta>|\sigma|, r>0$ and $|x|>3 r+R$ we obtain

$$
\mathbf{E}^{x}\left[e^{-\eta \tau_{r}^{(1)}}\right] \leq \int_{\bar{B}(0, r)} G_{1}^{\eta}(x-y) \mu_{r}^{\eta,(1)}(d y) \leq \sup _{y \in \bar{B}(0, r)} G_{1}^{\eta}(x-y) \mu_{r}^{\eta,(1)}(\bar{B}(0, r)) .
$$

By the local uniform comparability of the densities $p^{(1)}(t, x)$, for every $|x|>3 r+R$ we have

$$
\begin{aligned}
\sup _{y \in \bar{B}(0, r)} G_{1}^{\eta}(x-y) & \leq \int_{0}^{\infty} e^{-\eta t} \sup _{y \in \bar{B}(0, r)} p^{(1)}(t, x-y) d t \leq C_{17}^{\lceil 2 r\rceil} \int_{0}^{\infty} e^{-\eta t} p^{(1)}\left(t, x-x_{r}\right) d t \\
& =C_{17}^{2\lceil 2 r\rceil} \int_{0}^{\infty} e^{-\eta t} \inf _{y \in \bar{B}\left(x_{2 r}, r\right)} p^{(1)}(t, x-y) d t \leq C_{17}^{2\lceil 2 r\rceil} \inf _{y \in \bar{B}\left(x_{2 r}, r\right)} G_{1}^{\eta}(x-y) .
\end{aligned}
$$

Moreover, $\mu_{r}^{\eta-|\sigma|,(2)}(\bar{B}(0, r))=\mu_{B\left(x_{2 r}, r\right)}^{\eta-|\sigma|,(2)}\left(\bar{B}\left(x_{2 r}, r\right)\right)$. Putting together the estimates above and using (2), we finally obtain

$$
\begin{aligned}
& \mathbf{E}^{x}\left[e^{-\eta \tau_{r}^{(1)}}\right] \leq \mu_{r}^{\eta,(1)}(\bar{B}(0, r)) \sup _{y \in \bar{B}(0, r)} G_{1}^{\eta}(x-y) \\
& \quad \leq C_{17}^{2\lceil 2 r\rceil} \frac{\mu_{r}^{\eta,(1)}(\bar{B}(0, r))}{\mu_{r}^{\eta-|\sigma|,(2)}(\bar{B}(0, r))} \mu_{B\left(x_{2 r}, r\right)}^{\eta-|\sigma|,(2)}\left(\bar{B}\left(x_{2 r}, r\right)\right) \inf _{y \in \bar{B}\left(x_{2 r}, r\right)} G_{2}^{\eta-|\sigma|}(x-y) \\
& \quad \leq C_{17}^{2\lceil 2 r\rceil} \frac{\mu_{r}^{\eta,(1)}(\bar{B}(0, r))}{\mu_{r}^{\eta-|\sigma|,(2)}(\bar{B}(0, r))} \mathbf{E}^{x}\left[e^{\left.-(\eta-|\sigma|) \tau_{\bar{B}\left(x_{2 r}, r\right)}^{(2)}\right]}=C_{17}^{2\lceil 2 r\rceil} \frac{\mu_{r}^{\eta,(1)}(\bar{B}(0, r))}{\mu_{r}^{\eta-|\sigma|,(2)}(\bar{B}(0, r))} \mathbf{E}^{x-x_{2 r}}\left[e^{-(\eta-|\sigma|) \tau_{r}^{(2)}}\right],\right.
\end{aligned}
$$

where $\tau_{\bar{B}\left(x_{2 r}, r\right)^{c}}^{(2)}=\inf \left\{t>0: X_{t}^{(2)} \in \bar{B}\left(x_{2 r}, r\right)\right\}$.

Making use of the above results, we can now derive an upper bound for the function defined in (3.13) for symmetric jump-paring processes with Lévy measures which are light at infinity in the sense that

$$
\int_{\mathbf{R}^{d}}|x|^{2} \nu(d x)<\infty .
$$

Since in this case property (3.11) in general does not hold, we require instead that

$$
\begin{gathered}
\text { there exist } C_{17} \geq 1 \text { and } R>0 \text { such that } p(t, x) \leq C_{17} p(t, y) \\
\text { for every } t>0 \text { and }|x| \geq|y| \geq R \text { satisfying }|x-y| \leq 1 \text {. }
\end{gathered}
$$

This means that the transition probability densities in some sense inherit the properties (A1.1)(A1.2) of the Lévy kernel, which is a reasonable requirement on the processes with jump intensities described by (A1). Note also that due to condition (3.16) we do not impose (2.19), and only assume that the general estimate (2.23) holds. Unlike the pivotal assumptions (2.15)-(2.16), both (3.17) and (2.23) should be seen as only technical assumptions providing a framework to our study. Note that they can be verified efficiently for a substantial subclass of jump-paring Lévy processes. For instance, if $A \equiv a$ Id for some $a \geq 0$ and $\nu$ is a non-increasing radial function (i.e., $\left(X_{t}\right)_{t \geq 0}$ is an isotropic unimodal Lévy process), then (3.17) and (2.23) automatically hold [8]. For more general 
processes with jump intensities satisfying (A1) which are not non-increasing radial functions, they can be verified under a mild regularity assumption on the profile $g$ around zero (see Proposition 3.2 below).

Theorem 3.3. Let $\left(X_{t}\right)_{t \geq 0}$ be a Lévy process with Lévy-Khintchin exponent $\psi$ given by (2.1)-(2.2) such that Assumptions (A1)-(A3) are satisfied. Moreover, suppose that conditions (2.23), (3.16)(3.17) and (2.15)-(2.16) hold. Then for every $\eta>0$ and $r>0$ there exist $C_{19}=C_{19}(X, \eta, r)$ and $R=R(X, \eta, r)>r$ such that

$$
\mathbf{E}^{x}\left[e^{-\eta \tau_{\bar{B}(0, r)^{c}}}\right] \leq C_{19} \nu(x), \quad|x| \geq R .
$$

Proof. We define a family of Lévy measures $\left(\nu^{s}\right)_{s \geq 4}$ by $\nu^{s}(d x)=\nu^{s}(x) d x$, with

$$
\nu^{s}(x):= \begin{cases}\sup _{s / 4 \leq|y| \leq s} \nu(y) & \text { for } s / 4 \leq|x| \leq s, \\ \nu(x) & \text { otherwise. }\end{cases}
$$

Let $\left(X_{t}^{s}\right)_{t \geq 0}$ be a Lévy process determined by the Lévy-Khintchin exponent $\psi^{s}$ (defined as in (2.1), with Lévy triplet $\left.\left(0, A, \nu^{s}\right)\right)$. We also define the corresponding symmetrization $\Psi^{s}$ and profile function $H^{s}$ as in (2.3) and (2.4), respectively. Note that $\nu^{s}=\nu+\sigma$, where $\sigma$ is a finite nonnegative measure with density $\sigma(x)=\left(\sup _{s / 4 \leq|y| \leq s} \nu(y)-\nu(x)\right) \mathbf{1}_{\{s / 4 \leq|x| \leq s\}}$. Moreover,

$$
\int_{|x| / 2<|y|<|x|}|y|^{2} \nu(y) d y \geq\left(4 C_{5}\right)^{-1} g(|x|)|x|^{2}|\{y:|x| / 2<|y|<|x|\}|
$$

for $|x| \geq 1$. Thus, by (3.16), we have $\nu(x) \leq \nu^{s}(x) \wedge c_{1}|x|^{-d-2}$, for all $|x| \geq 1$ and $s \geq 4$. This and (2.4) immediately give

$$
c_{2} r^{2} \leq \Psi(r) \leq \Psi^{s}(r) \leq c_{3} H^{s}\left(\frac{1}{r}\right) \leq c_{4}\left(r^{2}+s^{-2}\right), \quad r \in(0,1], \quad s \geq 4,
$$

with constants $c_{2}, c_{3}, c_{4}$ independent of $s$ and $r$. For more clarity, we divide the remainder of the proof into four steps.

Step 1. In this step we estimate the function $K_{3}^{X^{s}}(r)$, the constants $C_{3}\left(X^{s}, r\right)$ given by (2.7), and the mean exit time from a ball for the process $\left(X_{t}^{s}\right)_{t \geq 0}$ with Lévy measure $\nu^{s}, s \geq 4$. As a consequence we also obtain general upper bounds for the corresponding functions $h_{1}, h_{2}$.

First notice that by (2.8), (2.10) and (3.19), we have

$$
\mathbf{E}^{0}\left[\tau_{B(0, r)}^{X^{s}}\right] \leq c_{5} r^{2} \quad \text { and } \quad C_{3}\left(X^{s}, r\right) \leq c_{6}\left(r^{-2}+s^{-2}\right),
$$

for every $r \geq 1$ and $s \geq 4$, with constants $c_{5}, c_{6}$ independent of $s$ and $r$. Moreover, we can also prove that there exists a constant $c_{7}$ (also uniform in $s$ and $r$ ) such that

$$
K_{3}^{X^{s}}(r) \leq c_{7} r^{4-d}\left(r^{-2}+r^{d} s^{-d-2}\right), \quad r \geq 8, \quad s \geq 4 .
$$

Indeed, by the second inequality in Proposition 3.1(1), the transition densities $p^{s}$ of $\left(X_{t}^{s}\right)_{t \geq 0}$ satisfy $p^{s}(t, x) \leq p(t, x)+t \sup _{z \in \mathbf{R}^{d}} \sigma(z), x \in \mathbf{R}^{d}, t>0$. Under assumption (2.23), this implies that $\sup _{|x| \geq r} p^{s}(t, x) \leq c_{8} t\left(\Psi(1 / r) r^{-d}+s^{-d-2}\right)$, for every $t>0, r \geq 1$ and $s \geq 4$, with the constant $c_{8}$ independent of $s, r, t$. Moreover, by [37, Lem. 5(a)], (A2) and (3.19), we obtain for $t>t_{b}$

$$
\int_{\mathbf{R}^{d}} e^{-t \psi^{s}(\xi)} d \xi \leq e^{-\left(t-t_{b}\right) \inf _{|z| \geq 1} \psi(z)} \int_{|\xi| \geq 1} e^{-t_{b} \psi(\xi)} d \xi+\int_{|\xi|<1} e^{-c_{9} t|\xi|^{2}} d \xi \leq \frac{c_{10}}{t^{d / 2}} \leq c_{11}\left(\Psi^{-1}\left(\frac{1}{t}\right)\right)^{d} .
$$

Therefore, (3.21) follows from Lemma 2.2 applied to $\left(X_{t}^{s}\right)_{t \geq 0}$, with $\Phi=\Psi$ and $\Theta=s^{-d-2}$. 
Next we take $\left(X_{t}^{s}\right)_{t \geq 0}$ with Lévy measure $\nu^{s}, s \geq 4$, and consider the functions $h_{1}, h_{2}$. Using the bounds (3.20)-(3.21), for every $r_{1} \geq 8, r_{2} \geq 2 r_{1}$ and $s \geq 4$ we obtain

$$
h_{1}\left(X^{s}, r_{1}, r_{2}\right) \leq K_{2}^{X^{s}}\left(r_{1}, r_{2}, \infty\right)\left[c_{12}\left(1+\left(\frac{r_{1}}{s}\right)^{2}\right)\left(1+\left(\frac{r_{1}}{s}\right)^{d+2}+\left(K_{2}^{X^{s}}\left(\frac{r_{1}}{4}, \frac{r_{1}}{2}, r_{1}\right)\right)^{2}\right)+1\right]
$$

and

$$
\begin{aligned}
r_{1}^{d} h_{2}\left(X^{s}, r_{1}\right) \leq \frac{c_{13}}{r_{1}^{2}}\left(1+\left(\frac{r_{1}}{s}\right)^{2}\right)\left[\left(1+\left(\frac{r_{1}}{s}\right)^{2}\right)\left(1+\left(\frac{r_{1}}{s}\right)^{d+2}+\left(K_{2}^{X^{s}}\left(\frac{r_{1}}{4}, \frac{r_{1}}{2}, r_{1}\right)\right)^{2}\right)\right. & \\
& \left.+\left(1 \vee\left(\frac{r_{1}}{s}\right)\right)^{d+2}\right]+c_{14} r_{1}^{d}\left(\frac{1}{r_{1}^{d+2}} \vee \frac{1}{s^{d+2}}\right),
\end{aligned}
$$

where the constants $c_{12}, c_{13}, c_{14}$ do not depend on $s, r_{1}$ and $r_{2}$. The function $K_{2}^{X^{s}}$ appearing in the above bounds still does depend on the specific form of $\nu^{s}$, i.e., on $s$.

Step 2. Now we choose a specific $s$ to obtain a suitable form of the function $K_{2}^{X^{s}}$ using the general upper bounds for the functions $h_{1}, h_{2}$ corresponding to $\left(X_{t}^{s}\right)_{t \geq 0}$ with Lévy measure $\nu^{s}$ in the previous step. Note that while in the previous step the constants $c_{12}, c_{13}, c_{14}$ are independent of $s$, and $r_{1}, r_{2}$ are such that $s \geq 4, r_{1} \geq 8$ and $r_{2} \geq 2 r_{1}$, from now on we assume that $s=r_{1}$ in (3.18).

By the definition of $\nu^{r_{1}}$ we see that $\nu^{r_{1}}(x)=\nu(x)$ for $|x|>r_{1}$ and $\nu^{r_{1}}(x)=\sup _{r_{1} / 4 \leq|z| \leq r_{1}} \nu(z)$ for $r_{1} / 4 \leq|x| \leq r_{1}$. With this, we obtain for $r_{1} \geq 8$ and $r_{2} \geq 2 r_{1}$ that

$$
K_{1}^{X^{r_{1}}}\left(r_{2}\right)=K_{1}^{X}\left(r_{2}\right), \quad K_{2}^{X^{r_{1}}}\left(r_{1}, r_{2}, \infty\right)=K_{2}^{X}\left(r_{1}, r_{2}, \infty\right) \quad \text { and } \quad K_{2}^{X^{r_{1}}}\left(\frac{r_{1}}{4}, \frac{r_{1}}{2}, r_{1}\right) \leq C_{5}^{2} .
$$

The latter bound is a consequence of the fact that for $|y| \leq r_{1} / 4$ and $r_{1} / 2 \leq|x|<r_{1}$ we have

$$
\nu^{r_{1}}(x-y)=\nu^{r_{1}}(x), \quad \frac{r_{1}}{4} \leq|x-y| \leq r_{1}
$$

and

$$
\nu^{r_{1}}(x-y)=\nu(x-y) \leq C_{5} g(|x-y|) \leq C_{5} g(|x|) \leq C_{5}^{2} \nu(x) \leq C_{5}^{2} \nu^{r_{1}}(x), \quad r_{1}<|x-y| \leq \frac{5 r_{1}}{4} .
$$

In particular, by (3.22)-(3.23) and the discussion of the function $K_{2}^{X^{r_{1}}}$ above, we arrive at

$$
h_{1}\left(X^{r_{1}}, r_{1}, r_{2}\right) \leq c_{15} K_{2}^{X}\left(r_{1}, r_{2}, \infty\right), \quad r_{1} \geq 8, \quad r_{2} \geq 2 r_{1},
$$

and

$$
r_{1}^{d} h_{2}\left(X^{r_{1}}, r_{1}\right) \leq \frac{c_{16}}{r_{1}^{2}}, \quad r_{1} \geq 8
$$

Note also that for any $r_{2} \geq 2 r_{1}$ and $r_{3}>r_{2}+r_{1}$ we have $K_{2}^{X^{r_{1}}}\left(r_{2}, r_{3}, \infty\right)=K_{2}^{X}\left(r_{2}, r_{3}, \infty\right)$.

Step 3. Let now $\kappa_{1} \geq 2$ and $\kappa_{2}<\infty$ be the parameters given by (2.15)-(2.16). From the construction made in the previous two steps we obtain that for every $\eta>0$ there is $r_{0}=r_{0}(\eta)$ such that for every $r_{1} \geq r_{0}$ there exists a Lévy process $\left(X_{t}^{r_{1}}\right)_{t \geq 0}$ with Lévy measure $\nu^{r_{1}}$ given by (3.18), $r_{2}=\kappa_{1} r_{1} \geq 2 r_{1}$ and $r_{3}>r_{2}+r_{1}$ for which

$$
2 d C_{5}^{4} h_{1}\left(X^{r_{1}}, r_{1}, r_{2}\right) K_{1}^{X^{r_{1}}}\left(r_{2}\right)+h_{2}\left(X^{r_{1}}, r_{1}\right)\left|B\left(0, r_{2}\right)\right| K_{2}^{X^{r_{1}}}\left(r_{2}, r_{3}, \infty\right)<\eta .
$$

We thus proved that for given $\eta>0$ there exists $r_{0}=r_{0}(\eta)$ such that for every Lévy process $\left(X_{t}^{r_{1}}\right)_{t \geq 0}$ with $r_{1} \geq r_{0}$ the assumption (3.7) of Lemma 3.2 is satisfied with $r_{1}, r_{2}$ and $r_{3}$. Hence for 
every $\eta>0$ there exists $r_{0}=r_{0}(\eta)$ such that for all $r_{1} \geq r_{0}$ and $R_{1}>0$ there exists a constant $c_{17}$ for which

$$
\mathbf{E}^{x}\left[e^{-\eta \tau_{\bar{B}}^{X_{1}}\left(0, R_{1}\right)^{c}}\right] \leq c_{17} \nu^{r_{1}}(x), \quad|x| \geq R_{2} .
$$

where $\tau_{\bar{B}\left(0, R_{1}\right)^{c}}^{X^{r_{1}}}=\inf \left\{t>0: X_{t}^{r_{1}} \in \bar{B}\left(0, R_{1}\right)\right\}$ and $R_{2}=\left(r_{1}+R_{1}\right) \vee r_{3}$.

Step 4. We now complete the proof of the claimed bound for the initial Lévy process with Lévy measure $\nu$. Let $\eta>0$ and $r_{0}=r_{0}(\eta / 2)$ be such that for every $r_{1} \geq r_{0}$ the estimate (3.24) holds for the process $\left(X_{t}^{r_{1}}\right)_{t \geq 0}$ with Lévy measure $\nu^{r_{1}}$ and $\eta / 2$ instead of $\eta$. Choose $r_{1}=r_{1}(\eta) \geq r_{0}$ such that $\left|B\left(0, r_{1}\right)\right| \sup _{r_{1} / 4 \leq|z| \leq r_{1}} \nu(z) \leq c_{18} r_{1}^{-2} \leq \eta / 2$ and observe that for such $r_{1}$ we have $\nu(x) \leq \nu^{r_{1}}(x)$, $x \in \mathbf{R}^{d} \backslash\{0\}$, and $\nu^{r_{1}}\left(\mathbf{R}^{d} \backslash\{0\}\right)-\nu\left(\mathbf{R}^{d} \backslash\{0\}\right) \leq \eta / 2$. This means that the assumptions of Proposition 3.1 are satisfied with $\nu^{(1)}=\nu, \nu^{(2)}=\nu^{r_{1}}$ and $\sigma=\nu^{r_{1}}-\nu$ for given $\eta$ and $|\sigma| \leq \eta / 2$. Thus by (3.14) and (3.24) we finally obtain

$$
\mathbf{E}^{x}\left[e^{-\eta \tau_{\bar{B}\left(0, R_{1}\right)^{c}}^{r_{1}}}\right] \leq c_{19} \mathbf{E}^{x-x_{2 R_{1}}}\left[e^{-(\eta / 2) \tau_{\bar{B}\left(0, R_{1}\right)^{c}}^{r_{1}}}\right] \leq c_{20} \nu^{r_{1}}\left(x-x_{2 R_{1}}\right), \quad|x| \geq\left(R_{2} \vee R\right)+3 R_{1},
$$

where $R$ comes from (3.17). The conclusion follows now from the fact that $\nu^{r_{1}}(x)=\nu(x)$ for $|x|>r_{1}$ and (A1.1)-(A1.2).

Recall that if $\left(X_{t}\right)_{t \geq 0}$ is an isotropic unimodal Lévy process, then both (3.17) and (2.23) hold. We conclude this section by a general sufficient condition on the profile $g$ around zero, which allows to extend this property to jump intensities as in (A1).

Proposition 3.2. Let $\left(X_{t}\right)_{t \geq 0}$ be a Lévy process determined by the Lévy-Khintchin exponent $\psi$ as in (2.1)-(2.2) with Lévy triplet $(0, A, \nu)$, such that $A=a \operatorname{Id}$ for some $a \geq 0$ and $\nu$ obeys (A1). Moreover, suppose that (3.16) is satisfied and there exist $\gamma_{1}, \gamma_{2} \in(0,2)$ and $C_{20}, C_{21}$ such that

$$
C_{20} \lambda^{-d-\gamma_{1}} g(r) \leq g(\lambda r) \leq C_{21} \lambda^{-d-\gamma_{2}} g(r), \quad \lambda \in(0,1], \quad r \in(0,1] .
$$

Then conditions (3.17) and (2.23) are satisfied. In particular, $\left(X_{t}\right)_{t \geq 0}$ is a symmetric jump-paring Lévy process, i.e., both of the remaining Assumptions (A2)-(A3) in Definition 2.1 hold.

Proof. Let $\psi_{\nu}(\xi)=\int_{\mathbf{R}^{d} \backslash\{0\}}(1-\cos (\xi \cdot z)) \nu(z) d z, \xi \in \mathbf{R}^{d}$. Denote the corresponding symmetrization of the characteristic exponent and the transition densities by $\Psi_{\nu}$ and $p_{\nu}$, respectively. Clearly, $\psi(\xi)=a|\xi|^{2}+\psi_{\nu}(\xi)$. Also, we denote by $p_{a}(t, x, y)=p_{a}(t, y-x)=(4 \pi a t)^{-d / 2} \exp \left(-|y-x|^{2} /(4 a t)\right)$ the transition densities of the diffusion part of $\left(X_{t}\right)_{t \geq 0}$, whenever $a>0$. It follows from (2.4) that $c_{1} \lambda^{\gamma_{1}} \Psi_{\nu}(r) \leq \Psi_{\nu}(\lambda r) \leq c_{2} \lambda^{\gamma_{2}} \Psi_{\nu}(r), \lambda, r \geq 1$. By this, [37, Lem. 5 (b)] and (A1.3), we see that both conditions (1.1) (a) and (b) in [37, Th. 1] are satisfied. Thus there exist $c_{3}, \theta, t_{0}>0$ such that for every $t \in\left(0, t_{0}\right]$

$$
p_{\nu}(t, x) \asymp c_{3}\left(h(t)^{-d} \mathbf{1}_{\{|x| \leq \theta h(t)\}}+\operatorname{tg}(|x|) \mathbf{1}_{\{|x| \geq \theta h(t)\}}\right), \quad \text { with } \quad h(t):=\frac{1}{\Psi_{\nu}^{-1}\left(\frac{1}{t}\right)} .
$$

In fact, by [37, Prop. 1] we may also assume that $p_{\nu}(t, x) \leq c_{3}\left(h(t)^{-d} \wedge t g(|x|)\right), t \in\left(0, t_{0}\right], x \in \mathbf{R}^{d}$.

We are now in the position to prove (3.17). We need to consider only the case $a>0$; for $a=0$ the proof is similar and simpler. Let first $t \in\left(0, t_{0}\right]$ and $|x| \geq|y|$. By (3.26), we have

$$
p(t, x)=p_{\nu}(t, \cdot) * p_{a}(t, \cdot)(x) \leq c_{3} \int_{\mathbf{R}^{d}}\left(h(t)^{-d} \wedge t g(|x-z|)\right) p_{a}(t, z) d z=c_{3} \int_{\mathbf{R}^{d}} k(t, x-z) p_{a}(t, z) d z,
$$

where $k(t, x):=h(t)^{-d} \wedge t g(|x|)$. Observe that for every fixed $t \in\left(0, t_{0}\right]$ both $k(t, \cdot)$ and $p_{a}(t, \cdot)$ are non-increasing radial functions, and the convolution of such functions preserves this property. Thus 
$\int_{\mathbf{R}^{d}} k(t, x-z) p_{a}(t, z) d z \leq \int_{\mathbf{R}^{d}} k(t, y-z) p_{a}(t, z) d z$ and hence, $p(t, x) \leq c_{3}^{2} p(t, y)$, for $|x| \geq|y|$ and $t \in\left(0, t_{0}\right]$. Let now $t>t_{0}$ and $|x| \geq|y| \geq 1,|x-y| \leq h\left(t_{0}\right) / 2$. By (3.26) we have

$$
\begin{aligned}
p(t, x) & =\int_{\mathbf{R}^{d}} \int_{\mathbf{R}^{d}} p_{\nu}\left(t_{0}, x-z-w\right) p_{\nu}\left(t-t_{0}, w\right) d w p_{a}(t, z) d z \\
\leq & c_{3} \int_{\mathbf{R}^{d}} \int_{\mathbf{R}^{d}}\left(h\left(t_{0}\right)^{-d} \wedge t_{0} g(|x-z-w|)\right) p_{\nu}\left(t-t_{0}, w\right) p_{a}(t, z) d w d z \\
\leq & c_{3}\left(\iint_{|y-z-w| \leq \theta h\left(t_{0}\right)} h\left(t_{0}\right)^{-d}+\iint_{|y-z-w| \geq \theta h\left(t_{0}\right)} t_{0} g(|x-z-w|)\right) p_{\nu}\left(t-t_{0}, w\right) p_{a}(t, z) d w d z .
\end{aligned}
$$

Notice that by (A1.1)-(A1.2) there exists a constant $c_{4}=c_{4}\left(t_{0}\right) \geq 1$ for which $g(|x-z-w|) \leq$ $c_{4} g(|y-z-w|)$ on the set $|y-z-w| \geq \theta h\left(t_{0}\right)$ and thus the sum of the above two integrals is bounded by $c_{3}^{2} c_{4} p(t, y)$. Condition (3.17) follows immediately.

We now prove $(2.23)$ for the density $p_{\nu}$ (i.e., when $\left.a=0\right)$. We already proved that $p_{\nu}(t, x) \leq$ $c_{3} t g(|x|), x \in \mathbf{R}^{d}, t \in\left(0, t_{0}\right]$. Since $g(r) r^{d} \leq c_{5} \Psi_{\nu}(1 / r), r>0$, condition (2.23) follows from this for $t \in\left(0, t_{0}\right]$. Therefore it suffices to consider only the case $t>t_{0}$. By [37, Lem. 5 (a)], (3.16), (3.25) and (2.4), we have $\psi_{\nu}(\xi) \geq c_{6} \Psi_{\nu}(|\xi|) \geq c_{7}\left(|\xi|^{2} \wedge|\xi|^{\gamma_{1}}\right), \xi \in \mathbf{R}^{d}$. With this, for $t>t_{0}$ we obtain

$$
\begin{aligned}
\int_{\mathbf{R}^{d}} e^{-t \psi_{\nu}(\xi)}|\xi| d \xi & =\int_{|\xi| \geq 1} e^{-t \psi_{\nu}(\xi)}|\xi| d \xi+\int_{|\xi|<1} e^{-t \psi_{\nu}(\xi)}|\xi| d \xi \\
& \leq e^{-c_{6}\left(t-t_{0}\right) \Psi_{\nu}(1)} \int_{|\xi| \geq 1} e^{-c_{7} t_{0}|\xi|^{\gamma_{1}}}|\xi| d \xi+\int_{|\xi|<1} e^{-c_{7} t|\xi|^{2}}|\xi| d \xi \\
& \leq c_{8}\left(e^{-c_{6} t \Psi_{\nu}(1)}+t^{-(d+1) / 2} \int_{\mathbf{R}^{d}} e^{-c_{7}|\xi|^{2}}|\xi| d \xi\right) \leq c_{9} t^{-(d+1) / 2} .
\end{aligned}
$$

Since $\Psi_{\nu}^{-1}(1 / t) \asymp 1 / \sqrt{t}, t>t_{0}$, we see that the assumption (3) of [36, Th. 1] is satisfied for $T=\left(t_{0}, \infty\right)$. Moreover, by the monotonicity and the doubling property of $\Psi_{\nu}$, we can also directly check that the remaining assumptions (1)-(2) of this theorem hold for $f(r):=\Psi_{\nu}(1 / r) r^{-d}$ and $\gamma=d$. Hence we get

$$
p_{\nu}(t, x) \leq c_{10}\left(\frac{t \Psi_{\nu}(1 /|x|)}{|x|^{d}}+t^{-d / 2} e^{-c_{11} \frac{|x|}{\sqrt{t}} \log \left(1+c_{11} \frac{|x|}{\sqrt{t}}\right)}\right), \quad x \in \mathbf{R}^{d} \backslash\{0\}, \quad t>t_{0} .
$$

It suffices to estimate the exponential term only. If $|x| \geq \sqrt{t}$, then

$$
t^{-d / 2} e^{-c_{11} \frac{|x|}{\sqrt{t}} \log \left(1+c_{11} \frac{|x|}{\sqrt{t}}\right)} \leq c_{12} t^{-d / 2}(\sqrt{t} /|x|)^{d+2}=c_{12} t /|x|^{d+2},
$$

for a constant $c_{12}>0$. On the other hand, for $|x| \leq \sqrt{t}$ we similarly have

$$
t^{-d / 2} e^{-c_{11} \frac{|x|}{\sqrt{t}} \log \left(1+c_{11} \frac{|x|}{\sqrt{t}}\right)} \leq t^{-d / 2} \leq t^{-d / 2}(\sqrt{t} /|x|)^{d+2}=t /|x|^{d+2},
$$

and thus finally obtain

$$
p_{\nu}(t, x) \leq c_{13} t\left(\frac{\Psi_{\nu}(1 /|x|)}{|x|^{d}}+\frac{\left(1 /|x|^{2}\right)}{|x|^{d}}\right), \quad x \in \mathbf{R}^{d} \backslash\{0\}, \quad t>t_{0} .
$$

Since by (2.4) we have $\Psi_{\nu}(r) \geq\left(c_{7} / c_{6}\right) r^{2}, r \in(0,1]$, this implies $(2.23)$ for $p_{\nu}$ when $t>t_{0}$. We thus completed the proof of (2.23) for $p_{\nu}$ (i.e., when $a=0$ ). Suppose now that $a>0$. We have for every $r>0,|x| \geq r$ and $t>0$, that

$$
p(t, x)=\left(\int_{|y-x| \geq r / 2}+\int_{|y-x|<r / 2}\right) p_{\nu}(t, x-y) p_{a}(t, y) d y \leq c_{14} t\left(\frac{\Psi_{\nu}(1 / r)}{r^{d}}+\frac{\left(1 / r^{2}\right)}{r^{d}}\right)+t^{-d / 2} e^{-\frac{r^{2}}{16 a t}} .
$$


Similarly as above, we can show that $t^{-d / 2} e^{-r^{2} /(16 a t)} \leq c_{15} t / r^{d+2}$. Since $\Psi(r) \asymp \Psi_{\nu}(r) \asymp r^{2}$, $r \in(0,1]$, we conclude that $(2.23)$ holds for $p$ as well.

Assumption (A2) follows since the integral $\int_{|\xi| \geq 1} e^{-c_{7} t_{0}|\xi|^{\gamma_{1}}}|\xi| d \xi$ is convergent, while (A3) holds by the bound $\sup _{|x| \geq r} p(t, x)<c_{16} t, r>0$, with $c_{16}=c_{16}(r)$, and the general estimate as in (2.22).

Note that in fact assumption (2.23) can be directly extended to the case when $\xi \cdot A \xi \geq C|\xi|^{2}, \xi \in \mathbf{R}^{d}$, for some $C>0$ (i.e., under the uniform ellipticity condition). The same should be true for (3.17). We also conjecture that the above proposition holds in a greater generality and the requirement (3.25) can be relaxed.

\section{Spatial decay of eigenfunctions}

\subsection{Decaying potentials and basic properties of eigenfunctions}

Now we turn to discussing the spatial decay properties of eigenfunctions of non-local Schrödinger operators presented in the Introduction. Except for the last subsection, in this part we consider decaying potentials in the following sense:

(A4) Let $V \in \mathcal{K}_{ \pm}^{X}$ be such that $V(x) \rightarrow 0$ as $|x| \rightarrow \infty$.

The number $\lambda \in \mathbf{R}$ is an eigenvalue of the non-local Schrödinger operator $H$ if there exists an eigenfunction $\varphi \in L^{2}\left(\mathbf{R}^{d}\right)$ such that

$$
H \varphi=\lambda \varphi, \quad \text { i.e. } T_{t} \varphi=e^{-\lambda t} \varphi, \text { for every } t>0 .
$$

The problem of existence and other properties of negative eigenvalues for non-local Schrödinger operators has been widely studied $[52,53,12,22,26,41]$.

For the reader's convenience we now recall a standard sufficient condition for the existence of negative bound states (eigenfunctions for negative eigenvalues) for non-local Schrödinger operators. The following proposition is a direct consequence of a basic result due to Weder [53, Ths 3.6-3.7] and standard facts on self-adjoint operators [49, Prop. 10.4 and 12.8], therefore we omit its proof. Recall that $L$ and $\mathcal{E}$ are defined by (2.5)-(2.6), and that $L^{p}+L_{\varepsilon}^{\infty}\left(\mathbf{R}^{d}\right), p \geq 1$, denotes the space of real-valued functions $V$ on $\mathbf{R}^{d}$ such that for every $\varepsilon>0$ there exist $V_{1, \varepsilon} \in L^{p}\left(\mathbf{R}^{d}\right)$ and $V_{2, \varepsilon} \in L^{\infty}\left(\mathbf{R}^{d}\right)$ with $\left\|V_{2, \varepsilon}\right\|_{\infty}<\varepsilon$ for which $V=V_{1, \varepsilon}+V_{2, \varepsilon}$.

Proposition 4.1. Let $H_{0}=-L$ be the pseudo-differential operator with symbol $\psi$ given by (2.1), and $V=V_{+}-V_{-}$be a Borel function on $\mathbf{R}^{d}$ satisfying the following properties:

(1) $V_{ \pm} \in L^{p}+L_{\varepsilon}^{\infty}\left(\mathbf{R}^{d}\right)$, for some $p \in[1, \infty)$

(2) $V_{-}$is relatively bounded with respect to $H_{0}$ with relative bound strictly less than 1 in quadratic form sense, i.e., there exist constants $C_{22} \in(0,1)$ and $C_{23} \geq 0$ such that

$$
\left\|V_{-}^{1 / 2} f\right\|_{2}^{2} \leq C_{22} \mathcal{E}(f, f)+C_{23}\|f\|_{2}^{2}, \quad f \in D(\mathcal{E}) .
$$

Then the non-local Schrödinger operator $H=H_{0}+V$ can be defined as a self-adjoint operator in form sense. Moreover, we have $\operatorname{Spec} H=\operatorname{Spec}_{\text {ess }} H \cup \operatorname{Spec}_{\mathrm{d}} H$, where $\operatorname{Spec}_{\mathrm{ess}} H=\operatorname{Spec}_{\mathrm{ess}} H_{0}=[0, \infty)$, Spec $_{\mathrm{d}} H \subset(-\infty, 0)$, and $\mathrm{Spec}_{\mathrm{d}} H$ consists of isolated eigenvalues of finite multiplicity whenever it is non-empty. In particular, if there exists $f \in D(\mathcal{E})$ such that

$$
\mathcal{E}(f, f)+\int_{\mathbf{R}^{d}} V(x) f^{2}(x) d x<0,
$$

then $\operatorname{Spec}_{\mathrm{d}} H \neq \emptyset$. 


\section{Remark 4.1.}

(1) It is known that (2.24) is equivalent to the property that $\lim _{\eta \rightarrow \infty}\left\|(-L+\eta \mathrm{Id})^{-1}|V|\right\|_{\infty}=0$ (see e.g. [25, Prop. 3.4]). Moreover, a standard argument based on Stein's interpolation theorem (see e.g. [42, Prop. 3.35]) gives that the latter property implies (4.2) with arbitrarily small $C_{22}$ (i.e., infinitesimal form boundedness of $V$ with respect to $H_{0}=-L$ ). In particular, for any decaying potential both of assumptions (1) and (2) in Proposition 4.1 hold.

(2) For decaying potentials the number of negative eigenvalues is bounded from above in many cases of interest, and bounds are given by variants of the Cwikel-Lieb-Thirring inequality; for the class of non-local Schrödinger operators with Bernstein functions of the Laplacian see [26]. Whenever (4.3) holds and the number of negative eigenvalues is finite, a unique ground state exists.

(3) Let $0 \leq V_{0} \in \mathcal{K}^{X}, V_{0} \neq 0$, and $V_{a, b}(x):=-a V_{0}(b x)$, with $a, b>0$. Notice that for any nonzero $f \in D(\mathcal{E})$ there are appropriate choices of $a, b$ for which $\mathcal{E}(f, f)+\int_{\mathbf{R}^{d}} V_{a, b}(x) f^{2}(x) d x<0$. In particular, if $V_{0}$ is a non-increasing radial function and (4.3) holds for $V_{a_{0}, b_{0}}$ with some $a_{0}, b_{0}$, then this extends to all $a>a_{0}$ and $b \in\left(0, b_{0}\right)$. Moreover, let $f_{r}$ and $\mu_{r}$ be a ground state and ground state eigenvalue, respectively, for an isotropic unimodal Lévy process with diffusion matrix $\|A\| \mathrm{Id}$ and radially non-increasing Lévy density $g(|x|)$, killed on leaving the ball $B(0, r), r>0$ (recall that $g$ is determined by (A1)). Denote the corresponding quadratic form by $\left(\mathcal{E}_{0}, D\left(\mathcal{E}_{0}\right)\right)$. Clearly, $\operatorname{supp} f_{r}=B(0, r),\left\|f_{r}\right\|_{2}=1$, and it is seen that $f_{r}$ is a non-increasing radial function [2, Def.1.3, Cor.2.3], see also [31, Lem.3.1]. Since $\mathcal{E}\left(f_{r}, f_{r}\right) \leq C_{5} \mathcal{E}_{0}\left(f_{r}, f_{r}\right)=C_{5} \mu_{r}$, we have

$$
\mathcal{E}\left(f_{r}, f_{r}\right)+\int_{\mathbf{R}^{d}} V_{a, b}(x) f_{r}^{2}(x) d x \leq C_{5} \mu_{r}-a \int_{B(0, r)} V_{0}(b x) f_{r}^{2}(x) d x \leq C_{5} \mu_{r}-a V_{0}(b r),
$$

for $r>0$. In many cases good approximations of $\mu_{r}$ are known (see, e.g., [14, 35]). With this, for given $C_{5}, a$ and $b$, we can settle if the right hand side is negative and even estimate its distance from zero. On the other hand, when the underlying Lévy process is recurrent and $V \not \equiv 0$ is a non-positive bounded potential with compact support, then a negative bound state exists [12, Th. V.1].

Example 4.1. The following decaying potentials are some possible choices and applications.

(1) Potential wells: Let $V(x)=-v(x)$ with a compactly supported, non-negative bounded Borel function $v \not \equiv 0$. Specifically, we can choose $V(x)=-a \mathbf{1}_{B(0,1)}(b x)$, for $a, b>0$.

(2) Coulomb-type potentials: Let $g$ in Assumption (A1) be such that $g(r)=r^{-d-\alpha}, r \in(0,1]$, for some $\alpha \in(0,2)$, and let $V(x)=-\left(a_{1}|x|^{-\beta_{1}} \wedge a_{2}|x|^{-\beta_{2}}\right)$, with $\beta_{1} \in(0, \alpha \wedge d], \beta_{2} \in\left[\beta_{1}, \infty\right)$ and $a_{1}, a_{2}>0$. Then $V \in \mathcal{K}^{X}$ can be directly checked by using (2.25) and [37, Th. 2].

(3) Yukawa-type potentials: Let $g$ in Assumption (A1) be as in (2) above and let $V(x)=$ $-\left(a_{1}|x|^{-\beta_{1}} \wedge a_{2}|x|^{-\beta_{2}} e^{-b|x|}\right)$, with $\beta_{1} \in(0, \alpha \wedge d], \beta_{2} \in\left[\beta_{1}, \infty\right)$ and $a_{1}, a_{2}, b>0$.

(4) Pöschl-Teller potential: This is the case of $V(x)=-a / \cosh ^{2}(b|x|)$ with $a, b>0$.

(5) Morse potential: This is the case of $V(x)=a\left(\left(1-e^{-b\left(|x|-r_{0}\right)}\right)^{2}-1\right)$ with $a, b, r_{0}>0$.

We will everywhere below assume that every eigenfunction $\varphi$ is normalized so that $\|\varphi\|_{2}=1$. Moreover, by the assumption $V \in \mathcal{K}_{ \pm}^{X}$ we have $T_{t}\left(L^{2}\left(\mathbf{R}^{d}\right)\right) \subset L^{\infty}\left(\mathbf{R}^{d}\right)$ and $T_{t}\left(L^{\infty}\left(\mathbf{R}^{d}\right)\right) \subset C_{\mathrm{b}}\left(\mathbf{R}^{d}\right)$ for every $t>t_{\mathrm{b}}$, compare Lemma 2.3. Therefore $\varphi=e^{\lambda t} T_{t} \varphi \in C_{\mathrm{b}}\left(\mathbf{R}^{d}\right)$, in particular, it makes sense to study pointwise estimates of $\varphi$. 
When $\lambda_{0}:=\inf \operatorname{Spec} H$ is an isolated eigenvalue (i.e., a ground state exists), by standard arguments based on Lemma 2.3 (4) and [45, Th. XIII.43] it follows that it is unique and the corresponding eigenfunction $\varphi_{0}$ has a strictly positive version, which will be our choice throughout. It is known that whenever a ground state at eigenvalue $\lambda_{0} \neq 0$ exists, $\varphi_{0}$ is the only non-negative eigenfunction of $H$ corresponding to a non-zero eigenvalue.

Below we will often use the following resolvent representation of eigenfunctions. Let $\lambda$ and $\varphi$ be such that $T_{t} \varphi(x)=e^{-\lambda t} \varphi(x)$ for every $x \in \mathbf{R}^{d}$ and $t>0$. Choose $\theta \in \mathbf{R}$ such that $\theta+\lambda>0$. By integrating on both sides of the equality

$$
e^{-(\theta+\lambda) t} \varphi(x)=\mathbf{E}^{x}\left[e^{-\int_{0}^{t}\left(\theta+V\left(X_{s}\right)\right) d s} \varphi\left(X_{t}\right)\right], \quad t>0, x \in \mathbf{R}^{d},
$$

we obtain

$$
\varphi(x)=(\theta+\lambda) \int_{0}^{\infty} \mathbf{E}^{x}\left[e^{-\int_{0}^{t}\left(\theta+V\left(X_{s}\right)\right) d s} \varphi\left(X_{t}\right)\right] d t, \quad x \in \mathbf{R}^{d} .
$$

Notice that by combining this with (2.27) applied to $f=\varphi$ for an arbitrary open set $D \subset \mathbf{R}^{d}$ and $x \in D$, we readily obtain

$$
\varphi(x)=(\theta+\lambda) \mathbf{E}^{x}\left[\int_{0}^{\tau_{D}} e^{-\int_{0}^{t}\left(\theta+V\left(X_{s}\right)\right) d s} \varphi\left(X_{t}\right) d t\right]+\mathbf{E}^{x}\left[\tau_{D}<\infty ; e^{-\int_{0}^{\tau_{D}}\left(\theta+V\left(X_{s}\right)\right) d s} \varphi\left(X_{\tau_{D}}\right)\right]
$$

which will be a key formula in what follows.

We close this subsection by showing that positive eigenfunctions (in particular, ground states) are bounded from below by $\nu$, no matter what the absolute value of $\lambda$ is. This fact provides a general reference point and justifies the question how far is the decay of eigenfunctions from the Lévy intensity of the given process. We also show that if (A1.2) or (A1.3) fails to hold, then such eigenfunctions cannot be bounded from above by $\nu$, even when the absolute value of $\lambda$ is large. In the next subsections we will study upper bounds of the eigenfunctions.

Theorem 4.1 (Lower bound and necessary condition for upper bound). Let $\left(X_{t}\right)_{t \geq 0}$ be a symmetric Lévy process with Lévy-Khintchin exponent satisfying (2.1)-(2.2), and let assumption (A4) hold. Suppose that $\varphi \in L^{2}\left(\mathbf{R}^{d}\right)$ is a positive eigenfunction at eigenvalue $\lambda \in \mathbf{R}$. Then the following hold.

(1) If (A1.1)-(A1.2) are satisfied, then for every $\delta>0$ there exists $r=r(V, \delta) \geq 1$ such that

$$
\varphi(x) \geq K \nu(x), \quad|x|>r+1,
$$

where $K:=\frac{1-e^{-(|\lambda|+\delta)}}{C_{5}^{2} C_{6}^{|r|+1}(|\lambda|+\delta)} \mathbf{P}^{0}\left(\tau_{B(0,1)}>1\right) \int_{B(0, r)} \varphi(z) d z$.

(2) Let (A1.1) be satisfied and consider the following two disjoint cases.

(i) (A1.2) holds and (A1.3) does not hold.

(ii) (A1.2) does not hold (and hence also (A1.3) does not hold).

Then in either of cases (i) and (ii) we have

$$
\limsup _{|x| \rightarrow \infty} \frac{\varphi(x)}{\nu(x)}=\infty
$$

Proof. Take any $\delta>0$. Note that by assumption (A4) there exists $r \geq 1$ such that $\sup _{|y| \geq r}|V(y)| \leq$ $\delta / 2$. Also, let $\theta=|\lambda|+\delta / 2$. With this, an application of (4.5) to $D=\bar{B}(0, r)^{c}$ gives for every $|x|>r$

$$
\varphi(x) \geq \mathbf{E}^{x}\left[\tau_{\bar{B}(0, r)^{c}}<\infty ; e^{-(\theta+\delta / 2) \tau_{\bar{B}(0, r)^{c}}} \varphi\left(X_{\tau_{\bar{B}(0, r)^{c}}}\right)\right] .
$$


Both assertions (1) and (2) follow now by direct application of Theorem 3.1 to the function $f(y)=$ $\varphi(y),|y| \leq r, f(y)=\mathbf{E}^{y}\left[\tau_{\bar{B}(0, r)^{c}}<\infty ; e^{-(|\lambda|+\delta) \tau_{\bar{B}(0, r)^{c}}} \varphi\left(X_{\tau_{\bar{B}(0, r)^{c}}}\right],|y|>r\right.$, noticing that it is regular $(X,|\lambda|+\delta)$-harmonic in $\bar{B}(0, r)^{c}$.

\subsection{Upper bound: cases of sufficiently low-lying and arbitrary negative eigenvalues}

In this subsection we state our main results on the upper bounds of eigenfunctions in their most general form. They are consequences of the estimates for harmonic functions obtained in Section 3 and will be illustrated and discussed in detail in the next subsection, where we analyze specific classes and examples of processes of interest.

The first result says that whenever an eigenvalue is sufficiently low-lying below zero with respect to the given jump-paring Lévy process, the corresponding eigenfunction is dominated by $\nu$ at infinity.

Theorem 4.2 (Low-lying negative eigenvalues). Let $\left(X_{t}\right)_{t \geq 0}$ be a Lévy process with LévyKhintchin exponent $\psi$ given by (2.1)-(2.2) and let Assumptions (A1)-(A4) hold. Denote

$$
\eta_{0}(X):=2 C_{5}^{4} h_{1}(X, 1,2) K_{1}^{X}(2)+h_{2}(X, 1)|B(0,2)| K_{3}^{X}(2,3, \infty) .
$$

Suppose that $\varphi \in L^{2}\left(\mathbf{R}^{d}\right)$ is an eigenfunction for an eigenvalue $\lambda \in\left(-\infty,-\eta_{0}\right)$. Then there exist $C_{25}=C_{25}(X, \lambda)$ and $R=R(X, \lambda)>0$ such that

$$
|\varphi(x)| \leq C_{25}\|\varphi\|_{\infty} \nu(x), \quad|x| \geq R .
$$

Proof. Choose $\theta>|\lambda|$. Let $\varepsilon>0$ be small enough such that $|\lambda|-\varepsilon>\eta_{0}$, and $r>0$ be large enough such that $\sup _{|y| \geq r}|V(y)| \leq \varepsilon$. Denote $\tau_{r}:=\tau_{\bar{B}(0, r)^{c}}$. By (4.5) applied to $D=\bar{B}(0, r)^{c}$, for every $|x|>r$ we have

$$
\begin{aligned}
|\varphi(x)| & \leq(\theta-|\lambda|) \mathbf{E}^{x}\left[\int_{0}^{\tau_{r}} e^{-(\theta-\varepsilon) t}\left|\varphi\left(X_{t}\right)\right| d t\right]+\mathbf{E}^{x}\left[\tau_{r}<\infty ; e^{-\int_{0}^{\tau_{r}}\left(\theta+V\left(X_{s}\right)\right) d s}\left|\varphi\left(X_{\tau_{r}}\right)\right|\right] \\
& \leq\|\varphi\|_{\infty}\left(\frac{\theta-|\lambda|}{\theta-\varepsilon}+\mathbf{E}^{x}\left[e^{-(\theta-\varepsilon) \tau_{r}}\right]\right) .
\end{aligned}
$$

Thus by taking the limit $\theta \rightarrow|\lambda|$ we obtain

$$
|\varphi(x)| \leq\|\varphi\|_{\infty} \mathbf{E}^{x}\left[e^{-(|\lambda|-\varepsilon) \tau_{r}}\right], \quad|x|>r .
$$

To complete the proof it remains to apply Lemma 3.2 to the function $f$ as in (3.13) which is $(X, \eta)$ harmonic in $\bar{B}(0, r)^{c}$ with $\eta=|\lambda|-\varepsilon$. Indeed, by (4.6) its assumptions are now satisfied with $r_{1}=1$, $r_{2}=2$ and $r_{3}=3$.

Note that in fact in the above theorem it suffices to assume that (A3) holds for $R \leq 1$ only.

The next result gives sufficient conditions according to a slow or fast decay of the Lévy intensity under which the eigenfunctions corresponding to arbitrary eigenvalues $\lambda<0$ are bounded above at infinity by $\nu$. Recall the discussion of the specific conditions below as done before Theorems 3.2-3.3.

Theorem 4.3 (Arbitrary negative eigenvalues). Let $\left(X_{t}\right)_{t \geq 0}$ be a Lévy process with LévyKhintchin exponent $\psi$ given by (2.1)-(2.2) and let Assumptions (A1)-(A4) hold. Suppose that $\varphi \in L^{2}\left(\mathbf{R}^{d}\right)$ is an eigenfunction at the eigenvalue $\lambda<0$. Consider the following cases.

(1) Lévy intensities with slow decay at infinity: conditions (3.11) and (2.19) hold.

(2) Lévy intensities with fast decay at infinity: conditions (3.16)-(3.17), (2.23) and (2.15)-(2.16) hold. 
In both cases (1) and (2) above there exist $C_{24}=C_{24}(X, \lambda)$ and $R=R(X, \lambda)>0$ such that

$$
|\varphi(x)| \leq C_{24}\|\varphi\|_{\infty} \nu(x), \quad|x| \geq R .
$$

Proof. Let $\lambda$ be as in the assumption. Take $\varepsilon>0$ small enough so that $|\lambda|-\varepsilon>0$, and $r>0$ large enough such that $\sup _{|y| \geq r}|V(y)| \leq \varepsilon$. Denote $\tau_{r}:=\tau_{\bar{B}(0, r)^{c}}$. By the same argument as in the proof of Theorem 4.2 above we get

$$
|\varphi(x)| \leq\|\varphi\|_{\infty} \mathbf{E}^{x}\left[e^{-(|\lambda|-\varepsilon) \tau_{r}}\right], \quad|x|>r .
$$

The claimed bound now follows for cases (1) and (2) by an application of Theorems 3.2 and 3.3 with $\eta=|\lambda|-\varepsilon$, respectively.

Recall that whenever $\left(X_{t}\right)_{t \geq 0}$ is an isotropic unimodal jump-paring Lévy process in $\mathbf{R}^{d}, d \geq 3$, conditions (2.19), (2.23), (3.17) automatically hold, and the assumptions above dividing into specific subclasses reduce to (3.11) in case (1), and to (3.16) and the basic conditions (2.15)-(2.16) in case (2). As it will be seen below, assumptions (2.15)-(2.16) are essential and sharp in the sense that for a large class of processes they actually provide a necessary and sufficient condition for the ground state to be comparable to the Lévy density $\nu$ at infinity (see discussion preceding Proposition 4.2).

\subsection{Specific cases}

Throughout this subsection we assume that $\lambda<0$. As it follows from the results above, the behaviour of the eigenfunctions at infinity significantly depends on the relative position of the corresponding eigenvalue from the edge of the continuous spectrum and on the decay properties of the Lévy intensity. To illustrate this in some explicit detail, consider the specific choice

$$
\psi(\xi)=a|\xi|^{2}+\int(1-\cos (\xi \cdot z)) \nu(z) d z, \quad \xi \in \mathbf{R}^{d},
$$

of the characteristic exponent (2.1), where $a \geq 0, \nu(d x)=\nu(x) d x$ is such that $\nu\left(\mathbf{R}^{d} \backslash\{0\}\right)=\infty$, $\nu(x)=\nu(-x)$, and there is a non-increasing function $g:(0, \infty) \rightarrow(0, \infty)$ such that

$$
C_{26} g(|x|) \leq \nu(x) \leq C_{27} g(|x|), \quad x \in \mathbf{R}^{d} \backslash\{0\},
$$

with constants $C_{26} \in(0,1]$ and $C_{27} \in[1, \infty)$. Moreover, whenever $C_{26}=C_{27}=1$ (i.e., $\nu$ is a non-increasing radial function), we impose the regularity condition

$$
g(r) \geq \frac{C_{28}}{r^{d}}, \quad r \in(0,1],
$$

on the small jumps with a constant $C_{28}$. On the other hand, when $C_{26}<1$ or $C_{27}>1$, we assume that (3.25) holds. In the proofs below we will often use the fact that (4.9) and (3.25) imply $\liminf |\xi| \rightarrow \infty \psi(\xi) / \log |\xi|>0$, which guarantees that assumption (A2) holds. Indeed, observe that by $[37$, Lem. 5 (a)], (2.4) and (4.8),

$$
\psi(\xi) \asymp \Psi(|\xi|) \geq C_{1} H(1 /|\xi|) \geq C_{1} a|\xi|^{2}+C_{1} C_{26} \int_{1 /|\xi|<|y|<1} g(|y|) d y, \quad|\xi|>1 .
$$

From this we see that $\psi(\xi) \geq C\left(a|\xi|^{2}+\log |\xi|\right)$ and $\psi(\xi) \geq C\left(a|\xi|^{2}+|\xi|^{\gamma_{1}}\right),|\xi|>1$, with some $C>0$ under (4.9) and (3.25), respectively. In particular, $p(t, 0)=\int_{\mathbf{R}^{d}} e^{-t \psi(\xi)} d \xi<\infty$, for sufficiently large $t>0$ and (A2) holds. Clearly, when $a>0$, the same is true even without (4.9) and (3.25). 
Corollary 4.1 (Polynomially decaying Lévy intensities). Let $\left(X_{t}\right)_{t \geq 0}$ be a Lévy process with Lévy-Khintchin exponent $\psi$ given by (4.7)-(4.8). Also, if (4.8) holds with $C_{26}=C_{27}=1$, then assume (4.9), if it holds with other values of constants, then assume (3.25). Let the profile $g$ satisfy

$$
g(r)=r^{-d-\delta}, \quad r \geq 1, \text { with } \delta>0 .
$$

If Assumption (A4) holds and $\varphi \in L^{2}\left(\mathbf{R}^{d}\right)$ is an eigenfunction corresponding to the eigenvalue $\lambda<0$, then there exist $C_{29}=C_{29}(X, \lambda)$ and $R=R(X, \lambda) \geq 1$ such that

$$
|\varphi(x)| \leq C_{29}\|\varphi\|_{\infty}|x|^{-d-\delta}, \quad|x| \geq R .
$$

Moreover, if $\varphi=\varphi_{0}$ is a ground state, then $\varphi_{0}(x) \asymp|x|^{-d-\delta},|x| \geq R$.

Proof. First observe that (A1.1) is assumed by default, and the remaining conditions (A1.2)-(A1.3) easily follow from (4.10). Assumption (A2) is a consequence of (4.9) or (3.25), and (2.19) follows from (2.21) and (2.23) as in Lemma 2.2. Indeed, by assuming (4.10) and by directly checking that

$$
\Psi(r) \asymp\left\{\begin{array}{ll}
r^{\delta} & \text { for } \quad 0<\delta<2, \\
r^{2} \log (1 / r) & \text { for } \delta=2, \\
r^{2} & \text { for } \delta>2,
\end{array} \quad r \in(0,1],\right.
$$

condition (2.21) with $\Phi=\Psi$ can be established similarly as in (3.27). Moreover, whenever $C_{26}=$ $C_{27}=1,(2.23)$ holds automatically for all $r>0$. Otherwise, due to (3.25) and (4.10), the same holds by Proposition 3.2. Specifically, when $\delta>2$, (2.23) follows directly from the statement of this result, and when $\delta \in(0,2]$, this can be obtained by following through the argument in the proof. Assumption (A3) holds as well. Secondly, notice that (3.11) immediately follows from (4.10). The proof of the upper bound can be completed by an application of Theorem 4.3 in case (1). The lower bound on $\varphi_{0}$ follows directly by Theorem 4.1 .

Remark 4.2. The assumptions of the above theorem cover the cases when

$$
g(r)=r^{-d-\gamma} \mathbf{1}_{\{r \in(0,1]\}}+r^{-d-\delta} \mathbf{1}_{\{r \geq 1\}}, \quad \text { with } \quad \gamma \in[0,2) \text { and } \delta>0,
$$

and $\nu(x)=g(|x|)$ for $\gamma \in[0,2)$, or more generally, $\nu(x) \asymp g(|x|)$ for $\gamma \in(0,2)$. For $a=0$, the first class includes some important cases of subordinate Brownian motion and isotropic unimodal Lévy processes like the isotropic $\alpha$-stable process $(\gamma=\delta=\alpha \in(0,2))$, their mixtures of stability indices $\alpha_{1}, \ldots, \alpha_{n}\left(\gamma=\min _{i} \alpha_{i}, \delta=\max _{i} \alpha_{i}\right)$, geometric $\alpha$-stable process $(\gamma=0, \delta=\alpha \in(0,2))$, layered $\alpha$-stable processes $(\gamma=\alpha \in(0,2), \delta>2)$, and many others. All of the above examples can also be considered with a non-zero Brownian component $(a>0)$, which covers a wide class of jump-diffusions. The second case, where $\nu$ is not a strictly radial function, allows a variety of polynomial-type processes like symmetric stable-like, strictly stable, and other Lévy processes with jump intensities comparable to the cases of isotropic processes as above.

Next we consider the case when the Lévy intensity is lighter than polynomial but heavier than exponential at infinity.

Corollary 4.2 (Sub-exponentially decaying Lévy intensities). Let $\left(X_{t}\right)_{t \geq 0}$ be a Lévy process with Lévy-Khintchin exponent $\psi$ given by (4.7)-(4.8). Moreover, if (4.8) holds with $C_{26}=C_{27}=1$, then assume (4.9), if it holds with other values of constants, then assume (3.25). Also, let the profile g satisfy

$$
g(r)=e^{-c r^{\beta}} r^{-\delta}, \quad r \geq 1, \quad \text { with } c>0, \beta \in(0,1) \text { and } \delta \geq 0 .
$$


If Assumption (A4) holds and $\varphi \in L^{2}\left(\mathbf{R}^{d}\right)$ is an eigenfunction at the eigenvalue $\lambda<0$, then there exist $C_{30}=C_{30}(X, \lambda)$ and $R=R(X, \lambda) \geq 1$ such that

$$
|\varphi(x)| \leq C_{30}\|\varphi\|_{\infty} e^{-c|x|^{\beta}}|x|^{-\delta}, \quad|x| \geq R .
$$

Moreover, if $\varphi=\varphi_{0}$ is a ground state, then $\varphi_{0}(x) \asymp e^{-c|x|^{\beta}}|x|^{-\delta},|x| \geq R$.

Proof. The upper bound follows from Theorem 4.3 in case (2). Assumptions (A1.1)-(A1.2) hold by default, (A1.3) by [37, Prop. 2], and (A2) follows from $\liminf |\xi| \rightarrow \infty \psi(\xi) / \log |\xi|>0$, which is the case under assumptions (4.9) or (3.25). Also, (3.16) is immediate and implies (2.21). With this, whenever $C_{26}=C_{27}=1$, assumptions (3.17), (2.23) and (A3) are satisfied automatically, otherwise they follow from Proposition 3.2. Thus it suffices to verify the remaining conditions (2.15)-(2.16).

First we show that there exist constants $c_{1}, c_{2}$ such that for every $s_{2}>2 s_{1} \geq 2$

$$
K_{1}^{X}\left(s_{2}\right) \leq c_{1} s_{2}^{d-\delta-\beta} e^{-c(1-\beta) s_{2}{ }^{\beta}} \quad \text { and } \quad K_{2}^{X}\left(s_{1}, s_{2}, \infty\right) \leq c_{2} e^{\frac{c \beta s_{1}}{\left(s_{2}-s_{1}\right)^{1-\beta}}} .
$$

For the first bound, observe that for every $x, y \in \mathbf{R}^{d}, y \neq 0, x \neq y$, we have by Lagrange's theorem

$$
\begin{aligned}
|y|^{\beta}+|x-y|^{\beta} & =(|y| \vee|x-y|)^{\beta}+(|y| \wedge|x-y|)^{\beta} \\
& \geq(|y|+|x-y|)^{\beta}-\frac{\beta(|y| \wedge|x-y|)}{(|y| \vee|x-y|)^{1-\beta}}+(1-\beta)(|y| \wedge|x-y|)^{\beta}+\beta(|y| \wedge|x-y|)^{\beta} \\
& \geq|x|^{\beta}-\frac{\beta(|y| \wedge|x-y|)}{(|y| \vee|x-y|)^{1-\beta}}+(1-\beta)(|y| \wedge|x-y|)^{\beta}+\frac{\beta(|y| \wedge|x-y|)}{(|y| \wedge|x-y|)^{1-\beta}} \\
& \geq|x|^{\beta}+(1-\beta)(|y| \wedge|x-y|)^{\beta} .
\end{aligned}
$$

Thus, by (4.11) and (2.12), we obtain

$$
K_{1}^{X}\left(s_{2}\right) \leq c_{3} \int_{|y|>s_{2}} e^{-c(1-\beta)|y|^{\beta}}|y|^{-\delta} d y
$$

which gives the required bound. A similar argument gives $|x|^{\beta}-|x-y|^{\beta} \leq \beta s_{1} /\left(s_{2}-s_{1}\right)^{1-\beta}$ for all $|x| \geq s_{2}$ and $|y| \leq s_{1}$, and thus

$$
g(|x-y|)=g(|x|) \frac{g(|x-y|)}{g(|x|)} \leq e^{\frac{c \beta s_{1}}{\left(s_{2}-s_{1}\right)^{1-\beta}}}\left(1+\frac{s_{1}}{s_{2}-s_{1}}\right)^{\delta} g(|x|), \quad|x| \geq s_{2}, \quad|y| \leq s_{1},
$$

which yields the claimed bound for $K_{2}^{X}$. In particular, for $\kappa_{1} \geq 2$ we have

$$
K_{1}^{X}\left(\kappa_{1} s_{1}\right) K_{2}^{X}\left(s_{1}, \kappa_{1} s_{1}, \infty\right) \leq c_{1} c_{2}\left(\kappa_{1} s_{1}\right)^{d-\delta-\beta} e^{-c\left((1-\beta) \kappa_{1}^{\beta}-\frac{\beta}{\left(\kappa_{1}-1\right)^{1-\beta}}\right) s_{1} \beta}, \quad s_{1} \geq 1, \quad \kappa_{1} \geq 2,
$$

which implies (2.15) for every $\kappa_{1}>2 \vee(\beta /(1-\beta))^{1 / \beta}$. Condition (2.16) with $\kappa_{2} \geq c_{2}$ easily follows from the upper bound of $K_{2}^{X}$ established above. The lower bound on $\varphi_{0}$ is a direct consequence of Theorem 4.1.

Remark 4.3. With some lengthy but straightforward computations we can show that the decay of $\nu(x)$ at infinity interpolating between the polynomial and sub-exponential cases such as $|x|^{-\log |x|}$ leads to similar bounds of eigenfunctions in terms of $\nu(x)$ as in Corollaries 4.1-4.2. The same applies for $\nu(x) \asymp e^{-|x| / \log |x|},|x| \rightarrow \infty$. Note that this decay rate is even closer to the strictly exponential case than in Corollary 4.2. The details are left to the reader. 


\subsection{Phase transition in the decay rates and strongly tempered Lévy intensities}

As seen in the previous subsection, the fall-off of the ground state is determined by the Lévy intensity $\nu$ as long as this decays strictly sub-exponentially. We now describe a qualitative change ("phase transition") in the decay rate behaviour. We show that if $|\lambda|$ is small with respect to the spectral edge of the free process and $\nu$ decreases exponentially, then the decay rate of $\varphi_{0}$ is slower than $\nu$, with essential contribution of $\lambda$. However, when $|\lambda|$ is large enough, the fall-off is again dominated by $\nu$ as long as the basic jump-paring condition (A1.3) holds (recall that this implies (A1.2)). In particular, this partly includes the exponential case. On the other hand, when (A1.2) or (A1.3) fails, then as proved in Theorem 4.1, the fall-off of $\varphi_{0}$ is slower than of $\nu$, no matter how large $\left|\lambda_{0}\right|$ is. This class includes not only super-exponentially decaying Lévy intensities, but also cases of exponentially decaying Lévy intensities for which the jump paring condition does not hold, so the exponential case falls on the dividing line in a peculiar way.

First we consider the exponential case.

Corollary 4.3 (Exponentially decaying Lévy intensities). Let $\left(X_{t}\right)_{t \geq 0}$ be a Lévy process with Lévy-Khintchin exponent $\psi$ given by (4.7)-(4.8) with $a=0$ and let Assumption (A4) hold. Moreover, let (3.25) be satisfied and assume

$$
g(r)=e^{-c r} r^{-\delta}, \quad r \geq 1, \quad \text { with } c>0 \text { and } \delta \geq 0 .
$$

Then we have the following:

(1) If a ground state at eigenvalue $\lambda_{0}<0$ exists, then there exist $C_{31}>0$ and $R>0$ such that

$$
\varphi_{0}(x) \geq C_{31} e^{-c|x|}|x|^{-\delta}, \quad|x| \geq R .
$$

(2) If $\delta>(d+1) / 2$ (i.e. (A1.3) holds), then for every eigenfunction $\varphi$ corresponding to the eigenvalue $\lambda \in\left(-\infty,-\eta_{0}\right)$ there exists a constant $C_{32}>0$ and $R>0$ such that

$$
|\varphi(x)| \leq C_{32}\|\varphi\|_{\infty} e^{-c|x|}|x|^{-\delta}, \quad|x| \geq R
$$

In particular, if $\delta>(d+1) / 2$ and $\lambda_{0} \in\left(-\infty,-\eta_{0}\right)$, then $\varphi_{0}(x) \asymp e^{-c|x|}|x|^{-\delta},|x| \geq R$.

(3) If (3.25) holds with $\gamma_{1}=\gamma_{2}$ and a ground state at eigenvalue $\lambda_{0}<0$ exists, then there is a constant $C_{33}>c$ such that for every $\varepsilon>0$ there exist $C_{34}>0$ and $R>0$ for which

$$
\varphi_{0}(x) \geq C_{34}\left(e^{-C_{33} \sqrt{\left|\lambda_{0}\right|+\varepsilon}|x|} \vee e^{-c|x|}|x|^{-\delta}\right), \quad|x| \geq R .
$$

Proof. The assumptions (A1.1)-(A1.2), (A2) and (A3) follow by similar arguments as above. Assumption (A1.3) holds if and only if $\delta>(d+1) / 2$ [37, Prop. 2]. The lower bound in terms of $e^{-c|x|}|x|^{-\delta}$ in (1)-(3) is a direct consequence of Theorem 4.1. Similarly, the upper bound in (2) follows from Theorem 4.2. It suffices to show that if (3.25) holds with $\gamma_{1}=\gamma_{2}$, then $\varphi_{0}(x) \geq C_{34} e^{-C_{33} \sqrt{\left|\lambda_{0}\right|+\varepsilon}|x|}$, for sufficiently large $|x|$. Notice that we only need to consider the case $\left|\lambda_{0}\right|+\varepsilon \in(0,1]$. By (1.14) in [13, Th. 1.2], for every $\eta \in(0,1]$ and $|x| \geq 1$ we have

$$
G^{\eta}(x) \geq \int_{1}^{\infty} e^{-\eta t} p(t, x) d t \geq c_{1} \int_{1}^{\infty} e^{-\eta t} e^{-c_{2}\left(|x| \wedge \frac{|x|^{2}}{t}\right)} t^{-d / 2} d t \geq c_{1} e^{-c_{2} \sqrt{\eta}|x|} \int_{|x| / \sqrt{\eta}}^{\infty} e^{-\eta t} t^{-d / 2} d t
$$

with some $c_{1}>0$ and $c_{2} \geq c$. Moreover, $\int_{|x| / \sqrt{\eta}}^{\infty} e^{-\eta t} t^{-d / 2} d t \asymp e^{-\sqrt{\eta}|x|}|x|^{-d / 2}$. By the same argument as in the proof of Theorem 4.2 (with the converse inequalities) and using (3.15), for every $\varepsilon>0$ 
satisfying $\left|\lambda_{0}\right|+\varepsilon \in(0,1]$ there exist $r>0$ and $c_{3}=c_{3}(X, \eta, r)$ such that

$$
\begin{aligned}
\varphi_{0}(x) & \geq \inf _{|z| \leq r} \varphi_{0}(z) \mathbf{E}^{x}\left[e^{\left.-\left(\left|\lambda_{0}\right|+\varepsilon\right) \tau_{\bar{B}(0, r)^{c}}\right]}\right. \\
& \geq \inf _{|z| \leq r} \varphi_{0}(z) \mu_{B(0, r)}^{\left|\lambda_{0}\right|+\varepsilon}(\bar{B}(0, r)) \inf _{|y| \leq r} G^{\left|\lambda_{0}\right|+\varepsilon}(x-y) \geq c_{3} e^{-\left(1+c_{2}\right) \sqrt{\left|\lambda_{0}\right|+\varepsilon}|x|}|x|^{-d / 2}, \quad|x|>r+1 .
\end{aligned}
$$

Note that the condition $\gamma_{1}=\gamma_{2}$ in part (3) of the corollary is a technical assumption required only for an application of the large-time estimates of transition densities in [13].

The above result covers a large family of Lévy processes with jump intensities exponentially localized at infinity. Important examples to this class are relativistic $\alpha$-stable-like processes $(c=$ $\left.m^{1 / \alpha}, \delta=(d+1+\alpha) / 2, \alpha \in(0,2)\right)$ for which (A1.3) holds, and other processes with Lévy measures with tails for which (A1.3) does not hold, such as variance gamma-like (that is, geometric 2-stablelike) processes $(c=1, \delta=(d+1) / 2)$, and some Lamperti-type transformed stable processes $(c>0$, $\delta=0)$.

The next result, taken together with Corollaries 4.1, 4.2 and 4.3, shows that for a large subclass of jump-paring Lévy processes assumptions (2.15)-(2.16) give in fact a necessary and sufficient condition for the result in Theorem 4.3. In particular, for Lévy intensities $\nu$ such that $\nu(x) \asymp$ $e^{-c|x|^{\beta}}|x|^{-\delta}$ for $|x| \rightarrow \infty$, with $c>0, \beta \in(0,1], \delta \geq 0$, conditions (2.15)-(2.16) hold if and only if $\beta \in(0,1)$, which is equivalent to the property that $\varphi_{0} \asymp \nu$. Recall that when the process is outside the jump-paring class (i.e., $\beta=1$ and $\delta \in[0,(d+1) / 2]$ or $\beta>1$ in the case above), $\varphi_{0}$ decays slower than $\nu$.

Proposition 4.2. Let $\left(X_{t}\right)_{t \geq 0}$ be a Lévy process with Lévy-Khintchin exponent $\psi$ given by (4.7)(4.8) such that (3.25) and (4.12) hold. Whenever $\delta>(d+1) / 2$, conditions (3.16)-(3.17) and (2.23) hold. However, there exist constants $C_{35}, C_{36}$ such that for every $s_{2}>2 s_{1} \geq 2$ we have

$$
K_{1}^{X}\left(s_{2}\right) \geq C_{35} s_{2}{ }^{(d+1) / 2-\delta} \quad \text { and } \quad K_{2}^{X}\left(s_{1}, s_{2}, \infty\right) \geq C_{36} e^{c s_{1}} .
$$

In particular, (2.15)-(2.16) fail to hold for any $\kappa_{1} \geq 2$ and $\kappa_{2}<\infty$.

Proof. Note that (4.12) yields (3.16), while (3.17), (2.23) are consequences of Proposition 3.2. The first bound on $K_{1}^{X}$ follows by the estimates in [37, Prop. 2, p. 22]. To prove the second bound, let $x_{s_{2}}=\left(s_{2}, 0, \ldots, 0\right), y_{s_{1}}=\left(s_{1}, 0, \ldots, 0\right)$ and observe that

$$
g\left(\left|x_{s_{2}}-y_{s_{1}}\right|\right)=g\left(\left|x_{s_{2}}\right|\right) \frac{g\left(\left|x_{s_{2}}-y_{s_{1}}\right|\right)}{g\left(\left|x_{s_{2}}\right|\right)} \geq e^{c s_{1}}\left(\frac{s_{2}}{s_{2}-s_{1}}\right)^{\delta} g\left(\left|x_{s_{2}}\right|\right) \geq e^{c s_{1}} g\left(\left|x_{s_{2}}\right|\right),
$$

which gives

$$
K_{2}^{X}\left(s_{1}, s_{2}, \infty\right) \geq \frac{\nu\left(x_{s_{2}}-y_{s_{1}}\right)}{\nu\left(x_{s_{2}}\right)} \geq c_{1} e^{c s_{1}}, \quad s_{2}>2 s_{1} \geq 2 .
$$

In particular, it is directly seen that none of the conditions (2.15) and (2.16) can hold for any $\kappa_{1} \geq 2$ and $\kappa_{2}<\infty$.

As seen from Theorem 4.2 and Corollary 4.3, a sufficiently low-lying ground state eigenvalue $\lambda_{0}<0$ secures good decay properties of the corresponding eigenfunction. An important application of this type of spectral information is in mathematical physics in studies of the stability of quantum systems with nuclear repulsion. For the case of one-electron quasi-relativistic atoms with Coulomb interaction see [17]. 
By using the following simple and intuitively clear criterion, we can easily settle when is $\lambda_{0}<0$ small enough in comparison with the magnitude of the potential. Denote by $\mu_{r}>0$ the principal eigenvalue of the semigroup of the process $\left(X_{t}\right)_{t \geq 0}$ killed on exiting the ball $B(0, r), r>0$.

Proposition 4.3. Let $\left(X_{t}\right)_{t \geq 0}$ be a Lévy process with Lévy-Khintchin exponent $\psi$ given by (2.1)(2.2) and let Assumption (A4) hold. Suppose that a ground state at the eigenvalue $\lambda_{0}<0$ exists. Then for every $r>0$ we have

$$
\lambda_{0} \leq \sup _{|y| \leq 2 r} V_{+}(y)-\inf _{|y| \leq 2 r} V_{-}(y)+\mu_{r} .
$$

Proof. For every $r>0$ denote $c_{1}=c_{1}(r):=1 /|B(0,2 r)|$. We have for all $t>0$ that

$$
\begin{aligned}
c_{1} \int_{B(0, r)}\left(\mathbf{E}^{x}\left[\tau_{B(x, r)}>t ; e^{-\int_{0}^{t} V\left(X_{s}\right) d s}\right]\right)^{2} d x & \leq \int_{\mathbf{R}^{d}}\left(\mathbf{E}^{x}\left[e^{-\int_{0}^{t} V\left(X_{s}\right) d s} \sqrt{c_{1}} \mathbf{1}_{B(0,2 r)}\left(X_{t}\right)\right]\right)^{2} d x \\
& \leq\left\|T_{t}\right\|_{2,2}^{2}=e^{-2 \lambda_{0} t} .
\end{aligned}
$$

For the paths starting at $x \in B(0, r)$ and satisfying $\tau_{B(x, r)}>t$ we have

$$
\int_{0}^{t} V\left(X_{s}\right) d s \leq t\left(\sup _{|y| \leq 2 r} V_{+}(y)-\inf _{|y| \leq 2 r} V_{-}(y)\right) \text {. }
$$

Hence we get

$$
c_{1}|B(0, r)|\left(\mathbf{P}^{0}\left(\tau_{B(0, r)}>t\right)\right)^{2} e^{-2 t\left(\sup _{|y| \leq 2 r} V_{+}(y)-\inf _{|y| \leq 2 r} V_{-}(y)\right)} \leq e^{-2 \lambda_{0} t}, \quad t>0, r>0 .
$$

Since $\mathbf{P}^{0}\left(\tau_{B(0, r)}>t\right) \geq c_{2} e^{-\lambda_{r} t}$ for all $t>0$ with the same constant $c_{2}=c_{2}(r)$, the above estimate gives

$$
2 \lambda_{0} t \leq 2 t\left(\sup _{|y| \leq 2 r} V_{+}(y)-\inf _{|y| \leq 2 r} V_{-}(y)\right)+2 \lambda_{r} t-\log \left[c_{1} c_{2}^{2}|B(0, r)|\right], \quad t>0, r>0 .
$$

Dividing on both sides by $2 t$ and taking the limit $t \rightarrow \infty$ the claim follows.

Corollary 4.4 (Super-exponentially decaying Lévy intensities). Let $\left(X_{t}\right)_{t \geq 0}$ be a Lévy process with Lévy-Khintchin exponent $\psi$ given by (4.7)-(4.8) with $a=0$. Moreover, let (3.25) with $\gamma_{1}=\gamma_{2}$ be satisfied and assume

$$
g(r)=e^{-c r^{\beta}} r^{-\delta}, \quad r \geq 1, \quad \text { with } c>0, \quad \beta>1 \text { and } \delta \geq 0 .
$$

If assumption (A4) holds and a ground state at eigenvalue $\lambda_{0}<0$ exists, then there is a constant $C_{37}>0$ such that for every $\varepsilon>0$ there exist $C_{38}>0$ and $R>0$ for which

$$
\varphi_{0}(x) \geq C_{38} e^{-C_{37} \sqrt{\left(\left|\lambda_{0}\right|+\varepsilon\right) \wedge 1}|x|(\log |x|)^{(\beta-1) / \beta}}, \quad|x| \geq R .
$$

Proof. By the lower estimate in (1.17) of [13, Th. 1.2], for sufficiently large $|x|$

$$
G^{\eta}(x) \geq \int_{1}^{|x|} e^{-\eta t} p(t, x) d t \geq c_{1} e^{-c_{2}\left(|x|(\log |x|)^{\frac{\beta-1}{\beta}}\right)} \int_{1}^{|x|} e^{-\eta t} t^{-d / 2} d t, \quad \eta>0,
$$

and

$$
\left.G^{\eta}(x) \geq \int_{\frac{|x|}{\sqrt{\eta}(\log |x|)^{\beta /(\beta-1)}}}^{\infty} e^{-\eta t} p(t, x) d t \geq c_{1} e^{-c_{2} \sqrt{\eta}\left(|x|(\log |x|)^{\frac{\beta-1}{\beta}}\right.}\right) \int_{\frac{|x|}{\sqrt{\eta}(\log |x|)^{\beta /(\beta-1)}}}^{\infty} e^{-\eta t} t^{-d / 2} d t,
$$

whenever $\eta \in(0,1]$. With this, the result follows by a similar argument as in Corollary 4.3(3).

Before closing this subsection we make a point about the mechanism behind the phase transition. 
Remark 4.4 (Phase transition in the decay rates). Denote by $\nu_{1}(d x)=\nu(x) \mathbf{1}_{\{|x| \geq 1\}} d x$ the Lévy measure of the compound Poisson process part, which we denote by $\left(\bar{X}_{t}\right)_{t \geq 0}$, in the Lévy-Itô decomposition of $\left(X_{t}\right)_{t \geq 0}$. Recall that the subsequent jumps $J_{1}, J_{2}, J_{3}, \ldots$ of $\left(\bar{X}_{t}\right)_{t \geq 0}$ are independent, identically distributed random variables on a common probability space $(\bar{\Omega}, \overline{\mathbf{P}})$, distributed by the probability measure $\bar{\nu}_{1}(d x)=\nu_{1}(d x) / \nu_{1}\left(\mathbf{R}^{d}\right)$. Observe that for a symmetric jump-paring process $\left(X_{t}\right)_{t \geq 0}$ we have by (A1.3) that

$$
\overline{\mathbf{P}}\left(\left|J_{1}+J_{2}\right|>r\right) \leq c_{1} \overline{\mathbf{P}}\left(\left|J_{1}\right|>r\right), \quad \text { for large } r>0,
$$

with some $c_{1}>0$. Obviously, the smallness conditions (2.15)-(2.16) are actually governed by the jump properties of $\left(\bar{X}_{t}\right)_{t \geq 0}$. On the level of the specific Lévy measures with "nice" enough profiles discussed in detail in the previous two subsections we see that the transition in the ground state fall-off rates occurs exactly when the decay rate of $\nu$ changes in leading order from strictly subexponential to exponential. In particular, the fall-off is driven by $\nu$ as long as

$$
\lim _{|x| \rightarrow \infty} \frac{\log \nu(x)}{|x|}=\lim _{|x| \rightarrow \infty} \frac{\log \bar{\nu}_{1}(x)}{|x|}=0 .
$$

One can conjecture that this qualitative change in the decay rates is strongly related to a change in the mechanism which makes the long jumps of the process occur. For simplicity, consider just the $d=1$ case. We can make the following comparison with the concept of subexponential distributions in probability [21]. It can be checked that for all the specific profiles discussed in Subsection 4.3 the measures $\bar{\nu}_{1}(d x)$ are cases of subexponential distributions, i.e., $\overline{\mathbf{P}}\left(\left|J_{1}+J_{2}\right|>r\right) \approx 2 \overline{\mathbf{P}}\left(\left|J_{1}\right|>r\right)$ as $r \rightarrow \infty$. Taken along with the general property $\overline{\mathbf{P}}\left(\left|J_{1}\right| \vee\left|J_{2}\right|>r\right) \approx 2 \overline{\mathbf{P}}\left(\left|J_{1}\right|>r\right)$, this implies $\overline{\mathbf{P}}\left(\left|J_{1}+J_{2}\right|>r\right) \approx \overline{\mathbf{P}}\left(\left|J_{1}\right| \vee\left|J_{2}\right|>r\right)$ as $r \rightarrow \infty$, which means that a double jump $J_{1}+J_{2}$ is larger than a large $r$ when either $J_{1}$ or $J_{2}$ is larger than $r$. It is much less likely that both $J_{1}, J_{2}$ are less than $r$, but they are large enough so that their sum exceeds $r$. This property extends to arbitrarily long sequences of jumps. When $\left(X_{t}\right)_{t \geq 0}$ is a jump-paring process but $\bar{\nu}_{1}(d x)$ is not subexponential, this is no longer true. From (4.14) we see that the ratio $\overline{\mathbf{P}}\left(\left|J_{1}+J_{2}\right|>r\right) / \overline{\mathbf{P}}\left(\left|J_{1}\right|>r\right)$ is bounded above for large $r$, but we only have $\liminf _{r \rightarrow \infty} \overline{\mathbf{P}}\left(\left|J_{1}+J_{2}\right|>r\right) / \overline{\mathbf{P}}\left(\left|J_{1}\right|>r\right)>2$. This gives $\liminf _{r \rightarrow \infty} \overline{\mathbf{P}}\left(\left|J_{1}+J_{2}\right|>r\right) / \overline{\mathbf{P}}\left(\left|J_{1}\right| \vee\left|J_{2}\right|>r\right)>1$ which means that the above described mechanism weakens. This can be seen as an increase of the capacity of the process to fluctuate through multiple smaller jumps rather than exceedingly large single jumps, which improves as the tail of $\bar{\nu}_{1}(d x)$, and thus of $\nu$, gets lighter.

\subsection{Upper bound for confining potentials}

Finally we show that by using Lemma 3.2 one of our recent results on eigenfunction estimates in [34] can be recovered. In this brief subsection we consider confining potentials in the sense of the following condition:

(A5) Let $V \in \mathcal{K}_{ \pm}^{X}$ be such that $V(x) \rightarrow \infty$ as $|x| \rightarrow \infty$.

Note that under Assumption (A5) the operators $T_{t}: L^{2}\left(\mathbf{R}^{d}\right) \rightarrow L^{2}\left(\mathbf{R}^{d}\right), t>0$, are compact, which follows by standard arguments based on approximation of $T_{t}, t \geq 2 t_{\mathrm{b}}$, in terms of compact operators [33, Lem. 3.2]. Clearly, compactness extends to all $t>0$ by the spectral theorem. The theory of operator semigroups implies then that there exists an orthonormal basis in $L^{2}\left(\mathbf{R}^{d}\right)$ consisting of the eigenfunctions $\varphi_{n}$ given by $T_{t} \varphi_{n}=e^{-\lambda_{n} t} \varphi_{n}, t>0, n \geq 0$, and the spectrum of $H$ consists only of eigenvalues $\lambda_{0}<\lambda_{1} \leq \lambda_{2} \leq \ldots \rightarrow \infty$ of finite multiplicity each. All $\varphi_{n}$ are bounded continuous functions, and the ground state $\varphi_{0}$ has a strictly positive version. 
The following result has been obtained first in [34, Th. 2.1]. For the sake of completeness, we show that in our present framework it can be obtained without jump estimates.

Theorem 4.4. Let $\left(X_{t}\right)_{t \geq 0}$ be a Lévy process with Lévy-Khintchin exponent $\psi$ given by (2.1)-(2.2) such that Assumptions (A1)-(A3) and (A5) hold. Suppose that $\varphi$ is an eigenfunction corresponding to the eigenvalue $\lambda \in \mathbf{R}$. Then there exist $C_{39}=C_{39}(X, V)>0$ and $R=R(X, V)>0$ such that

$$
|\varphi(x)| \leq C_{39}\|\varphi\|_{\infty} \nu(x), \quad|x|>R .
$$

Proof. Choose $\theta \in \mathbf{R}$ such that $\theta+\lambda>0$ and let $r>0$ be large enough so that $\kappa:=\inf _{|y| \geq r} V(y)>$ $\left(\eta_{0}+\lambda\right) \vee 0$, where $\eta_{0}$ is given by (4.6). Denote $\tau_{r}:=\tau_{\bar{B}(0, r)^{c}}$. Similarly as in Theorem 4.2, an application of (4.5) to $D=\bar{B}(0, r)^{c}$ gives for every $|x| \geq r+1$ that

$$
\begin{aligned}
|\varphi(x)| & \leq(\theta+\lambda) \mathbf{E}^{x}\left[\int_{0}^{\tau_{r}} e^{-(\theta+\kappa) t}\left|\varphi\left(X_{t}\right)\right| d t\right]+\mathbf{E}^{x}\left[\tau_{r}<\infty ; e^{-\int_{0}^{\tau_{r}}\left(\theta+V\left(X_{s}\right)\right) d s}\left|\varphi\left(X_{\tau_{r}}\right)\right|\right] \\
& \leq\|\varphi\|_{\infty}\left(\frac{\theta+\lambda}{\theta+\kappa}+\mathbf{E}^{x}\left[e^{-(\theta+\kappa) \tau_{r}}\right]\right) .
\end{aligned}
$$

We complete the proof by taking $\theta=-\lambda+\delta$ with $\delta>0$. Then by taking the limit $\delta \downarrow 0$ and by application of Lemma 3.2 with $\eta=\kappa-\lambda>\eta_{0}$ the claim follows.

Note that the above result is a key step in deriving sharp bounds for eigenfunctions for confining potentials [34, Th. 2.3-2.4].

Acknowledgements. We thank the Referee for a careful reading of the manuscript.

\section{References}

[1] S. Agmon: Lectures on Exponential Decay of Solutions of Second-Order Elliptic Equations: Bounds on Eigenfunctions of N-Body Schrödinger Operators, Princeton University Press, 1983, 2nd ed. 2014.

[2] F. J. Almgren, E. H. Lieb: Symmetric decreasing rearrangement is sometimes continuous, J. Amer. Math. Soc. $2,1989,683-773$.

[3] D. Applebaum: Lévy Processes and Stochastic Calculus, Cambridge University Press, 2nd ed., 2008.

[4] R. Bañuelos: Intrinsic ultracontractivity and eigenfunction estimates for Schrödinger operators, J. Funct. Anal. 100, 1991, 181-206.

[5] J. Bertoin: Lévy Processes, Cambridge University Press, 1996.

[6] R.M. Blumenthal, R. K. Getoor: Markov Processes and Potential Theory, Springer, 1968.

[7] K. Bogdan, T. Byczkowski: Potential theory for the $\alpha$-stable Schrödinger operator on bounded Lipschitz domain, Studia Math. 133 (1), 1999, 53-92.

[8] K. Bogdan, T. Grzywny, M. Ryznar: Density and tails for unimodal convolution semigroups, J. Funct. Anal. 266, 2014, 3543-3571.

[9] K. Bogdan et al: Potential Analysis of Stable Processes and its Extensions (ed. P. Graczyk, A. Stós), Lecture Notes in Mathematics 1980, Springer, 2009.

[10] K. Bogdan, T. Kumagai, M. Kwaśnicki: Boundary Harnack inequality for Markov processes with jumps, Trans. Amer. Math. Soc. 367(1), 2015, 477-517.

[11] R. Carmona: Pointwise bounds for Schrödinger eigenstates, Commun. Math. Phys. 62, 1978, 65-92.

[12] R. Carmona, W.C. Masters, B. Simon: Relativistic Schrödinger operators: asymptotic behaviour of the eigenfunctions, J. Funct. Anal. 91, 1990, 117-142.

[13] Z.-Q. Chen, P. Kim, T. Kumagai: Global heat kernel estimates for symmetric jump processes, Trans. Amer. Math. Soc. 363 (9), 2011, 5021-5055.

[14] Z.-Q. Chen, R. Song: Two sided eigenvalue estimates for subordinate processes in domains, J. Funct. Anal. 226, 2005, 90-113.

[15] Z.-Q. Chen, R. Song: General gauge and conditional gauge theorems, Ann. Probab. 30 (3), 2002, $1313-1339$.

[16] K.L. Chung, Z. Zhao: From Brownian Motion to Schrödinger's Equation, Springer, 1995.

[17] I. Daubechies, E. H. Lieb: One-electron relativistic molecules with Coulomb interaction, Commun. Math. Phys. 90, 1983, 497-510. 
[18] E.B. Davies, B. Simon: Ultracontractivity and heat kernels for Schrödingers operators and Dirichlet Laplacians, J. Funct. Anal. 59, 1984, 335-395.

[19] M. Demuth, J. A. van Casteren: Stochastic spectral theory for selfadjoint Feller operators. A functional analysis approach, Probability and its Applications, Birkhäuser Verlag, Basel, 2000.

[20] S.O. Durugo and J. Lörinczi: Spectral properties of the massless relativistic quartic oscillator, preprint, 2014

[21] P. Embrechts, C.M. Goldie, N. Veraverbeke: Subexponentiality and infinite divisibility, Z. Wahrscheinlichkeitstheorie verw. Gebiete 49, 1979, 335-347.

[22] R.L. Frank, E.H. Lieb, R. Seiringer, Hardy-Lieb-Thirring inequalities for fractional Schrödinger operators, J. Amer. Math. Soc. 21 (4), 2008, 925-950.

[23] P. Garbaczewski, V. Stephanovich, D. Kẹdzierski: Heavy-tailed targets and (ab)normal asymptotics in diffusive motion, Physica A 390, 2011, 990-1008.

[24] T. Grzywny: On Harnack inequality and Hölder regularity for isotropic unimodal Lévy processes, Potential Anal. 41, 2014, 1-29.

[25] T. Grzywny, K. Szczypkowski: Kato classes for Lévy processes, preprint, 2015, available at arXiv:1503.05747.

[26] F. Hiroshima, J. Lörinczi: Lieb-Thirring bound for Schrödinger operators with Bernstein functions of the Laplacian, Commun. Stoch. Anal. 6, 2012, 589-602.

[27] F. Hiroshima, T. Ichinose, J. Lörinczi: Path integral representation for Schrödinger operators with Bernstein functions of the Laplacian, Rev. Math. Phys. 24, 2012, 1250013.

[28] F. Hiroshima, T. Ichinose, J. Lörinczi: Probabilistic representation and fall-off of bound states of relativistic Schrödinger operators with spin 1/2, Publ. Res. Inst. Math. Sci., 49, 2013, 189-214.

[29] N. Ikeda, S. Watanabe: On some relations between the harmonic measure and the Lévy measure for a certain class of Markov processes, J. Math. Kyoto Univ. 2-1, 1961, 79-95.

[30] N. Jacob: Pseudo-Differential Operators and Markov Processes, vols. 1-3, Imperial College Press, $2003-2005$.

[31] K. Kaleta: Spectral gap lower bound for the one-dimensional fractional Schrödinger operator in the interval, Studia Math. 209, 2012, 267-287.

[32] K. Kaleta, T. Kulczycki: Intrinsic ultracontractivity for Schrödinger operators based on fractional Laplacians, Potential Anal. 33 (4), 2010, 313-339.

[33] K. Kaleta, J. Lörinczi: Fractional $P(\phi)_{1}$-processes and Gibbs measures, Stoch. Proc. Appl. 122, $2012,3580-3617$.

[34] K. Kaleta, J. Lörinczi: Pointwise eigenfunction estimates and intrinsic ultracontractivity-type properties of Feynman-Kac semigroups for a class of Lévy processes, Ann. Probab. 43 (3), 2015, 1350-1398.

[35] K. Kaleta, M. Kwaśnicki, J. Małecki: One-dimensional quasi-relativistic particle in the box, Rev. Math. Phys. 25 (8), 2013, 1350014.

[36] K. Kaleta, P. Sztonyk: Estimates of transition densities and their derivatives for jump Lévy processes, J. Math. Anal. Appl. 431 (1), 2015, 260-282.

[37] K. Kaleta, P. Sztonyk: Small time sharp bounds for kernels of convolution semigroups, J. Anal. Math, 2015, to appear, available at arXiv:1403.0912.

[38] P. Kim, R. Song, Z. Vondraček: Potential theory for subordinate Brownian motions revisited, Interdisciplinary Mathematical Sciences, Vol. 13, Stochastic Analysis and Applications to Finance, Essays in Honour of Jia-an Yan, September 2012.

[39] P. Kim, R. Song, Z. Vondraček: Potential theory of subordinate Brownian motions with Gaussian components, preprint, 2011, arXiv:1106.5858v2.

[40] V. Knopova, R.L. Schilling: A note on the existence of transition probability densities for Lévy processes, Forum Math. 25, 2013, 125-149.

[41] E.H. Lieb, R. Seiringer, The Stability of Matter in Quantum Mechanics, Cambridge University Press, 2009.

[42] J. Lörinczi, F. Hiroshima, V. Betz: Feynman-Kac-Type Theorems and Gibbs Measures on Path Space. With Applications to Rigorous Quantum Field Theory, de Gruyter Studies in Mathematics 34, Walter de Gruyter, 2011; 2nd edition to appear in 2015.

[43] J. Lörinczi, J. Małecki: Spectral properties of the massless relativistic harmonic oscillator, J. Diff. Equations 253 , 2012, 2846-2871.

[44] W.E. Pruitt: The growth of random walks and Lévy processes, Ann. Probab. 9, 1981, 948-956.

[45] M. Reed, B. Simon: Methods of Modern Mathematical Physics, vol. 4, Academic Press, 1980.

[46] K.I. Sato: Lévy Processes and Infinitely Divisible Distributions, Cambridge University Press, 1999.

[47] R. Schilling, R. Song, Z. Vondraček: Bernstein Functions, Walter de Gruyter, 2010.

[48] R. Schilling: Growth and Hölder conditions for the sample paths of Feller processes, Probab. Theory Relat. Fields $112,1998,565-611$.

[49] K. Schmüdgen: Unbounded Self-adjoint Operators on Hilbert Space, Graduate Texts in Mathematics 265, Springer 2012 .

[50] B. Simon: Schrödinger semigroups, Bull. Amer. Math. Soc. 7, 1982, 447-526.

[51] B. Simon: Functional Integration and Quantum Physics, 2nd ed., AMS Chelsea Publishing, 2004. 
[52] R.A. Weder: Spectral properites of one-body relativistic spin-zero Hamiltonians, Ann. IHP A20(2), 1977, $211-220$.

[53] R.A. Weder: Spectral analysis of pseudodifferential operators, J. Funct. Anal. 20, 1975, 319-337.

[54] Z. Zhao: A probabilistic principle and generalized Schrödinger perturbations, J. Funct. Anal. 101, 1991, $162-176$.

Kamil Kaleta, Institute of Mathematics, University of Warsaw, ul. Banacha 2, 02-097 Warszawa and Faculty of Pure and Applied Mathematics, Wroceaw University of Science and Technology, Wyb. Wyspiańskiego 27, 50-370 Wroceaw, Poland

E-mail address: kamil.kaleta@pwr.edu.pl, kkaleta@mimuw.edu.pl

József Lőrinczi, Department of Mathematical Sciences, Loughborough University, Loughborough LE11 3TU, United Kingdom

E-mail address: J.Lorinczi@lboro.ac.uk 